OPEN ACCESS

Edited by:

Mingkai Li,

Fourth Military Medical University,

China

Reviewed by:

$\mathrm{Hu}$ Zhu,

Fujian Normal University, China

Ying Zhou,

Xi'an Medical University, China

*Correspondence:

Mona I. Shaaban

mona_ibrahem@mans.edu.eg

Specialty section:

This article was submitted to

Clinical Microbiology,

a section of the journal

Frontiers in Cellular and

Infection Microbiology

Received: 29 May 2021 Accepted: 15 September 2021 Published: 30 September 2021

Citation:

Naga NG, El-Badan DE, Rateb HS, Ghanem KM and Shaaban MI (2021)

Quorum Sensing Inhibiting

Activity of Cefoperazone and

Its Metallic Derivatives on

Pseudomonas aeruginosa.

Front. Cell. Infect. Microbiol. 11:716789.

doi: 10.3389/fcimb.2021.716789

\section{Quorum Sensing Inhibiting Activity of Cefoperazone and Its Metallic Derivatives on Pseudomonas aeruginosa}

\author{
Nourhan G. Naga ${ }^{1}$, Dalia E. El-Badan ${ }^{1}$, Heba S. Rateb ${ }^{2}$, Khaled M. Ghanem ${ }^{1}$ \\ and Mona I. Shaaban ${ }^{3 *}$ \\ ${ }^{1}$ Department of Botany and Microbiology, Faculty of Science, Alexandria University, Alexandria, Egypt, ${ }^{2}$ Department of \\ Pharmaceutical and Medicinal Chemistry, Pharmacy College, Misr University for Science and Technology, Cairo, Egypt, \\ ${ }^{3}$ Department of Microbiology and Immunology, Faculty of Pharmacy, Mansoura University, Mansoura, Egypt
}

The last decade has witnessed a massive increase in the rate of mortalities caused by multidrug-resistant Pseudomonas aeruginosa. Therefore, developing new strategies to control virulence factors and pathogenicity has received much attention. One of these strategies is quorum sensing inhibition (QSI) which was developed to control Pseudomonas infection. This study aims to validate the effect of one of the most used $\beta$-lactam antibiotics; cefoperazone (CFP) and its metallic-derivatives on quorum sensing (QS) and virulence factors of $P$. aeruginosa. Assessment of quorum sensing inhibitory activity of CFP, cefoperazone Iron complex (CFPF) and cefoperazone Cobalt complex (CFPC) was performed by using reporter strain Chromobacterium violaceum ATCC 12472. Minimal inhibitory concentration $(\mathrm{MIC})$ was carried out by the microbroth dilution method. The influence of sub-MICs (1/4 and 1/2 MICs) of CFP, CFPF and CFPC on virulence factors of $P$. aeruginosa was evaluated. Data was confirmed on the molecular level by RT-PCR. Also, molecular docking analysis was conducted to figure out the possible mechanisms of QSI. CFP, CFPF, and CFPC inhibited violacein pigment production of $C$. violaceum ATCC 12472. Sub-MICs of CFP (128- $256 \mu \mathrm{g} / \mathrm{mL})$, and significantly low concentrations of CFPC (0.5- 16 $\mu \mathrm{g} / \mathrm{mL})$ and CFPF $(0.5-64 \mu \mathrm{g} / \mathrm{mL})$ reduced the production of QS related virulence factors such as pyocyanin, protease, hemolysin and eliminated biofilm assembly by $P$. aeruginosa standard strains PAO1 and PA14, and P. aeruginosa clinical isolates Ps1, Ps2, and Ps3, without affecting bacterial viability. In addition, CFP, CFPF, and CFPC significantly reduced the expression of las/ and rhll genes. The molecular docking analysis elucidated that the QS inhibitory effect was possibly caused by the interaction with QS receptors. Both CFPF and CFPC interacted strongly with Lasl, LasR and PqsR receptors with a much high ICM scores compared to CFP that could be the cause of elimination of natural ligand binding. Therefore, CFPC and CFPF are potent inhibitors of quorum sensing signaling and virulence factors of $P$. aeruginosa.

Keywords: quorum sensing inhibition, cefoperazone, Pseudomonas aeruginosa, virulence factors, LasR, cefoperazone derivatives, cefoperazone cobalt complex, cefoperazone-iron complex 


\section{INTRODUCTION}

Pseudomonas aeruginosa is an encapsulated, Gram-negative, rod-shaped opportunistic pathogen that infects plants, animals, and human (Borges et al., 2018). P. aeruginosa can colonize critical body organs such as lungs, urinary tract, and kidney with fatal pathological effects especially on immunocompromised patients (Turkina and Vikström, 2019).

Treatment of $P$. aeruginosa infections by antibiotics is getting ineffective due to the worldwide spread of multi-drug resistant isolates. In addition, $P$. aeruginosa possesses diverse virulence factors including biofilm, pyocyanin, elastase, aminopeptidase, chitinase, protease, lipase, alginate, and hydrogen cyanide (Schuster and Greenberg, 2006). All these virulence triats assist the spreading and dissemination of Pseudomonas infection in different organs.

Pseudomonas virulence behaviors are synchronized by QS signaling system. Four fundamental QS pathways have been distinguished in $P$. aeruginosa. First, lasI/lasR system which uses 3-oxododecanoyl-L-homoserine lactone (3-oxo-C12-HSL) as an autoinducer. Second, rhlI/rhlR system which utilizes $\mathrm{N}$ butanoyl homoserine lactone (C4-HSL) as an autoinducer (Pesci et al., 1997). Both systems are linked to the third system, Pseudomonas quinolone signal (PqsABCDE/PqsR), which responses to 2-heptyl-3-hydroxy-4-quinolone autoinducer (Déziel et al., 2005; Dubern and Diggle, 2008). Fourth, the integrated quorum sensing, (AmbBCDE/IqsR), which uses the autoinducer 2-(2-hydroxyphenyl) thiazole-4-carbaldehyde (Lee \& Zhang, 2015; Rampioni et al., 2016). These four QS signaling systems are interconnected to regulate the expression of various virulence genes in P. aeruginosa (Parsek and Greenberg, 2000).

Inhibition of quorum sensing is one of the most promising approaches to counteract Pseudomonas virulence factors and associated pathogenic infection. QSI with different modes of action has been developed (Shaaban et al., 2019; Proctor et al., 2020). There are different natural and synthetic compounds that have been identified as QSIs. Natural compounds such as ascorbic acid (Vitamin C) (El-Mowafy et al., 2014), zingerone (Kim et al., 2015), curcumin (Tyagi et al., 2015), alginates (He et al., 2014), and $1 \mathrm{H}$-pyrrole-2-carboxylic acid (Hassan et al., 2016) have been identified as QSIs (Ahmed et al., 2019). Moreover, synthetic QSIs have been reported including halogenated furanones (Hentzer et al., 2002), benzothiazole (Gabr et al., 2015), isatin- $\beta$-thiocarbohydrazones (Gabr et al., 2018), and phenylalanine arginyl $\beta$-naphthylamide (El-Shaer et al., 2016).

In addition, various antimicrobial agents elicit anti-QS effects and eliminate QS-associated virulence factors. QSI activity of azithromycin (Tateda et al., 2001), doxycycline (Husain and Ahmad, 2013), and ciprofloxacin (Skindersoe et al., 2008) have been detected (Nalca et al., 2006). Moreover, $\beta$-lactams, ceftazidime, cefepime and imipenem have demonstrate QSI activity at sub-inhibitory concentrations (Skindersoe et al., 2008; El-Mowafy et al., 2017; Aleanizy et al., 2021).

CFP is a bactericidal third-generation cephalosporin possessing extended-spectrum activity against Gram-negative bacilli. Resistance to CFP has been developed where CFP was ineffective in the treatment of Pseudomonas infection. Thus, it is necessary to develop strategies to combat virulence factors without acquiring resistance. Masoud and coauthors have developed CFP metallic derivatives with antimicrobial activity against Gram-negative bacilli (Masoud et al., 2017). To our knowledge, QSI activity of CFP and its derivatives have not been evaluated. So, the aim of this study is to investigate the effect of CFP and its metallic complexes on QS circuits and virulence factors as a new strategy for combating Pseudomonas infection.

\section{MATERIALS AND METHODS}

\subsection{Bacterial Strains, Media, and Conditions} Chromobacterium violaceum ATCC 12472 reporter strain was used in the assay of QSI activity of CFP and its metallic complexes (McClean et al., 1997). The reporter strain was grown on Luria-Bertani (LB) media containing (tryptone, 10 $\mathrm{g} / \mathrm{L}$; yeast extract, $5 \mathrm{~g} / \mathrm{L}$; and $\mathrm{NaCl}, 10 \mathrm{~g} / \mathrm{L}$ ) at $\mathrm{pH}$ 7, and incubated at $28^{\circ} \mathrm{C}$ for 48 hours (Bertani, 2004). P. aeruginosa clinical isolates were isolated from urine samples and named Ps1, Ps2, and Ps3 according to the Helsinki declaration in handling, use and care of human subjects for medical research. Ethical approval was obtained from the Institutional Review Boards of Faculty of Medicine, Alexandria University, Egypt.

These clinical isolates were identified as $P$. aeruginosa according to laboratory biochemical standards. $P$. aeruginosa PAO1 and $P$. aeruginosa PA14 standard strains were also used for assessment of QSI effects of the tested chemical compounds

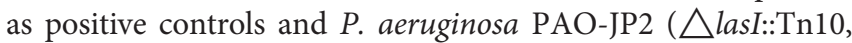
Tcr; $\triangle$ rhll::Tn501-2, Hgr) is a QS double-mutant strain and has been used as a negative control (Pearson et al., 1997). All $P$. aeruginosa strains were cultivated in LB media and incubated overnight at $37^{\circ} \mathrm{C}$.

\subsection{Cefoperazone and Metallic Derivatives}

CFP complexes with $\mathrm{FeCl}_{3} \cdot 6 \mathrm{H}_{2} \mathrm{O}, \mathrm{CoCl}_{2}, \mathrm{MnCl}_{2}, \mathrm{NiCl}_{2}$, and $\mathrm{CrCl}_{3}$ (Sigma Aldrich, USA) (Table 1) were prepared according to the method reported by Masoud and coauthors (Masoud et al., 2017). Each Metal chlorides (1 M) was dissolved in $40 \mathrm{~mL}$ ethanol and CFP (1 M) was dissolved in $40 \mathrm{~mL}$ doubledistilled water. Then, metal chloride ethanolic solutions and CFP solutions were mixed in a molar ratio of $1: 1$. Each reaction mixture was refluxed for 4 hours and left overnight. The formed precipitate was filtered and washed with $10 \mathrm{ml}$ ethanol $95 \% \mathrm{w} / \mathrm{v}$, and dried in a vacuum desiccator over

TABLE 1 | Inhibition of violet pigment of Chromobacterium violaceum ATCC 12472 by cefoperazone and its metallic derivatives.

\begin{tabular}{lllc}
\hline & Derivative & Code & Anti-QS zone (mm) \\
\hline Cefoperazone & - & CFP & 25 \\
& $\mathrm{FeCl}_{3} \cdot 6 \mathrm{H}_{2} \mathrm{O}$ & CFPF & 33 \\
$\mathrm{CoCl}_{2}$ & CFPC & 34 \\
$\mathrm{MnCl}_{2}$ & CFPM & - \\
& $\mathrm{NiCl}_{2}$ & CFPN & - \\
$\mathrm{CrCl}_{3}$ & CFPR & -
\end{tabular}


anhydrous $\mathrm{CaCl}_{2}$. The formed CFP metal complexes were confirmed by UV and thermal analysis (Masoud et al., 2017). Stock solutions of cefoperazone-metallic derivatives were prepared at a concentration $5 \mathrm{mg} / \mathrm{ml}$ in DMSO $(20 \% \mathrm{v} / \mathrm{v})$.

\subsection{QSI Assay of Cefoperazone and Metallic Complexes Using C. violaceum ATCC 12472}

C. violaceum ATCC 12472 reporter strain was used to determine the QSI activity of CFP and different complexes. The culture of C. violaceum was propagated at $28^{\circ} \mathrm{C}$ with $200 \mathrm{rpm}$ agitation in a shaking incubator for 48 hours. The double-layer culture plate method was performed where $15 \mathrm{~mL}$ of LB medium $(2 \% \mathrm{w} / \mathrm{v}$ agar) was poured into the plates $(9 \mathrm{~cm})$. Solidified LB plates were overlaid with $8 \mathrm{~mL}$ of soft LB medium (1\% w/v agar) containing $100 \mu \mathrm{L}$ of $C$. violaceum, and the plates were left to completely solidify. Wells were cut in the agar using a sterile cork borer (10 $\mathrm{mm}$ diameter). CFP and its derivative CFPC, and CFPF $(100 \mu \mathrm{L})$ at $1 / 2$ and $1 / 4$ MICs were loaded in the corresponding wells and the plates were incubated at $28^{\circ} \mathrm{C}$. The inhibition zone diameter of the violet color of violacein pigment around each well was measured after 24 hours (McClean et al., 1997; McLean et al., 2004). In each plate, DMSO was applied in one well as a control.

\subsection{Determination of Minimal Inhibitory Concentrations}

Compounds that showed inhibition of violacein pigment production were selected. The minimal inhibitory concentrations of the selected compounds; CFP, CFPC, and CFPF were determined using the microtitre plate assay method. Muller Hinton broth $(100 \mu \mathrm{L})$ was distributed in each well, $100 \mu \mathrm{L}$ of the tested compound was added to the first well. Two-fold serial dilution of the tested compound was performed in the subsequent wells to have serial dilutions of 512, 256, 128, $64,32,16,8,4,1$, and $0.5 \mu \mathrm{g} / \mathrm{mL}$. Each well was inoculated with 1 $\mathrm{X} 10^{5} \mathrm{CFU}$ of $P$. aeruginosa cultures Ps1, Ps2, Ps3, PAO1, PA14, and PAO-JP2. Wells containing media only and wells receiving media inoculated with the test strains were included in each plate as negative and positive controls, respectively. All plates were incubated overnight at $37^{\circ} \mathrm{C}$ and the microbial growth in each well was visually detected. The MIC was calculated as the lowest concentration that eliminated microbial growth compared to the positive control (CLSI, 2014). Sub-MICs of CFP, CFPC and CFPF were calculated, and the tested strains were propagated in the presence of sub-MICs of the tested compounds.

\subsection{The Effect of Sub-Inhibitory Concentrations on Bacterial Growth}

P. aeruginosa strains Ps1, Ps2, Ps3, PAO1, PA14 and PAO-JP2 were propagated in the presence of $1 / 2$ MICs of CFP, CFPC and CFPF. Control untreated strains were also cultivated under the same conditions. In brief, LB broth media $(25 \mathrm{~mL})$ supplemented with 1/2 MIC of the tested compounds CFP, CFPC and CFPF was inoculated with overnight culture of Ps1, Ps2, Ps3, PAO1, PA14 and PAO-JP2 to achieve 0.05 OD $600 \mathrm{~nm}$ at zero time. From each mixture, $1 \mathrm{~mL}$ was collected at different time intervals and the OD $600 \mathrm{~nm}$ was estimated. In addition, viable counts of treated and untreated $P$. aeruginosa strains Ps1, Ps2, Ps3, PAO1, PA14 and PAO-JP2 were estimated after 18 hours using the pour plate method. The collected samples were diluted 1:10 and $1 \mathrm{~mL}$ of each dilution was inoculated in LB agar $(15 \mathrm{~mL})$ and distributed in $9 \mathrm{~cm}$ plate. The plates were solidified and incubated at $37^{\circ} \mathrm{C}$ for 18 hours. The count of bacterial cells of each sample was calculated as the viable $P$. aeruginosa colonies $\mathrm{X}$ dilution factor and represented as $\mathrm{CFU} / \mathrm{mL}$ (Standards Australia, 1995).

\subsection{Effect of Sub-MICs of the Tested Compounds on Pseudomonas Virulence Factors}

CFP, CFPC and CFPF at 1/2 and 1/4 MICs did not exhibit any significant effect on microbial growth. So, they were assessed for their effect on different virulence factors of $P$. aeruginosa. The tested $P$. aeruginosa strains Ps1, Ps2, Ps3, PAO1, PA14, and PAO-JP2 (negative control) were propagated in the presence of $1 / 2$ and 1/4 MICs of CFP, CFPC, and CFPF (Musthafa et al., 2012). The untreated strains were also grown under the same conditions. Assay of different virulence factors was performed in the presence and absence of the tested compounds (El-Mowafy et al., 2017).

\subsubsection{Pyocyanin Assay}

Pyocyanin quantification was performed by using King's A broth media. Overnight culture of $P$. aeruginosa strains was inoculated into $5 \mathrm{~mL}$ of King's A media. Both untreated and treated cultures with sub-MICs (1/2 and 1/4 MICs) of CFP, CFPC, and CFPF were incubated at $37^{\circ} \mathrm{C}$ for 48 hours with shaking. Pyocyanin was extracted from the supernatant with $3 \mathrm{~mL}$ chloroform. The mixture was centrifuged at $3000 \mathrm{rpm}$ for $10 \mathrm{~min}$. Chloroform fractions were transferred to a new tube, then $1 \mathrm{~mL}$ of $0.2 \mathrm{M} \mathrm{HCL}$ was added, and the mixture was re-centrifuged for $5 \mathrm{~min}$ at 3000 $\mathrm{rpm}$. The absorbance of the aqueous layer was measured at OD $520 \mathrm{~nm}$. Pyocyanin concentration was calculated from the equation: pyocyanin concentration $(\mu \mathrm{g} / \mathrm{mL})=$ OD $520 \mathrm{~nm} \times$ 17.072 (He et al., 2014; Saurav et al., 2016).

\subsubsection{Total Protease Production}

The tested $P$. aeruginosa strains were inoculated in LB broth media supplemented with $1 / 2$ and $1 / 4$ MICs of CFP, CFPC, and $\mathrm{CFPF}$ and grown overnight at $37^{\circ} \mathrm{C}$. The untreated strains were also grown under the same conditions. Cells were centrifuged and proteolytic activity of the treated and untreated $P$. aeruginosa isolates was measured (Rossignol et al., 2008). Proteolytic activity was determined using skimmed milk assay technique (Skindersoe et al., 2008; El-Mowafy et al., 2017). The assay relies on determining the change in the turbidity of skimmed milk accompanying protease activity. Skimmed milk was freshly prepared by dissolving $1.25 \mathrm{gm}$ of skimmed milk in $100 \mathrm{~mL}$ sterile distilled water at $60^{\circ} \mathrm{C}$. Then, $0.5 \mathrm{~mL}$ of culture supernatant was added to $1 \mathrm{~mL}$ of the prepared skimmed milk, and the mixture was incubated at $37^{\circ} \mathrm{C}$ for 1 hour. The degree of clearance of skimmed milk was determined by measuring the 
turbidity at OD $600 \mathrm{~nm}$. The decrease in the OD $600 \mathrm{~nm}$ was indicated by the clearance of the skimmed milk with elevated proteolytic activity (El-Mowafy et al., 2014).

\subsubsection{Determination of Hemolysin Activity}

The tested $P$. aeruginosa strains were propagated in the presence of $1 / 2$ and $1 / 4 \mathrm{MICs}$ of CFP, CFPC, and CFPF overnight at $37^{\circ} \mathrm{C}$ (Musthafa et al., 2012). The untreated strains were also grown under the same conditions. Cells were centrifuged and the hemolytic activity of treated and untreated $P$. aeruginosa isolates was measured (Rossignol et al., 2008). RBCs (obtained from sheep) were washed three times with sterile physiological saline and re-suspended in tris-buffer $(\mathrm{pH} 7.4,0.025 \mathrm{M})$ to a final concentration of $2 \% \mathrm{v} / \mathrm{v}$. For hemolysin assay, $700 \mu \mathrm{L}$ of erythrocytes suspension was mixed with $500 \mu \mathrm{L}$ of treated and untreated cell-free supernatants and incubated for 2 hours at $37^{\circ} \mathrm{C}$. The suspension was centrifuged at $3000 \mathrm{rpm}$ for $10 \mathrm{~min}$ at $4^{\circ} \mathrm{C}$, and cell lysis was assessed by determining absorbance at $\mathrm{OD}$ $540 \mathrm{~nm}$.

\subsubsection{Quantification of Biofilm Formation Using Microtiter Plate Assay}

Flat bottomed polystyrene microtiter plates were used to evaluate slime production and biofilm formation. Overnight cultures of the tested $P$. aeruginosa strains were diluted with sterile LB broth to $0.5 \mathrm{McFarland}$. Treated and untreated cultures $(100 \mu \mathrm{L})$ were transferred to each well and the plates were incubated for 24 hours at $37^{\circ} \mathrm{C}$ for mature biofilm formation. The content of each well was aspirated using Pasteur pipette and each well was rinsed three times with $200 \mu \mathrm{l}$ of physiological saline. The plates were shaken to remove all non-adherent cells and the remaining attached bacteria were fixed with $150 \mu \mathrm{L}$ of absolute methanol for $15 \mathrm{~min}$, then, the plates were emptied and left to dry. Sessile cells bound to the wells were stained with $150 \mu \mathrm{L}$ of crystal violet $(1 \% \mathrm{v} / \mathrm{v})$ for $10 \mathrm{~min}$. Excess stain was rinsed off, and the plates were washed with water. The plates were air-dried and the dye bound to the wells was eluted with $150 \mu \mathrm{L}$ of $33 \%$ (v/v) glacial acetic acid (Adonizio et al., 2008). The absorbance was measured at OD $490 \mathrm{~nm}$ using a microtiter plate reader (Diaket, ELISA plate reader).

\subsection{Expression of QS Genes}

The effect of CFP, CFPC, and CFPF at sub-MICs on the expression of QS genes lasI and rhlI in P. aeruginosa PAO1 was assessed by RT-PCR. The untreated and treated PAO1 with 1/2 MIC of CFP, CFPC, and CFPF were propagated, and cells were collected at the middle of exponential phase. Total RNA was extracted by Triazole reagent (Sigma Chemicals, USA). Chloroform $(100 \mu \mathrm{L})$ was added to each sample, the tubes were incubated for 2-3 $\mathrm{min}$ at room temperature and centrifuged at $12.000 \mathrm{rpm}$ for $15 \mathrm{~min}$ at $4^{\circ} \mathrm{C}$. The aqueous phase was collected in RNase free Eppendorf tubes and chloroform step was repeated for complete purification of RNA. Isopropanol $(300 \mu \mathrm{L})$ was added to each tube, the tubes were mixed for $1 \mathrm{~min}$ and centrifuged at $12.000 \mathrm{rpm}$ for $15 \mathrm{~min}$ at $4^{\circ} \mathrm{C}$. The supernatant from the previous step was removed leaving RNA pellet. The pellet was washed twice with $1 \mathrm{ml}$ of ethanol $75 \% \mathrm{w} / \mathrm{v}$. The sample was gently mixed, and centrifuged at $10.000 \mathrm{rpm}$ for $5 \mathrm{~min}$ at $4^{\circ} \mathrm{C}$. The supernatant was discarded, and RNA pellet was air dried for 10-15 min. The concentration and the purity for each RNA sample were determined using NanoDrop (ND-1000 Spectrophotometer, NanoDrop Technologies, Wilmington, Delaware, USA).

Complementary DNA (cDNA) was synthesized using Quanti-Tect Reverse Transcription kit (Qiagen, Germany) according to the manufacturer's instructions. RT-PCR reaction mixture was composed of cDNA, $4 \mu \mathrm{L}$ of the 5X FIREPol Eva Green, qPCR Mix (Solis Bio- Dyne, Estonia), 2 nM of each primer (Table 2), and RNAase free water to final volume $20 \mu \mathrm{L}$. RT-PCR was performed as follows: one cycle at $95^{\circ} \mathrm{C}$ for $15 \mathrm{~min}$, followed by 40 cycles each cycle programmed as denaturation at $95^{\circ} \mathrm{C}$ for $15 \mathrm{~s}$, annealing for $30 \mathrm{~s}$ and extension at $72^{\circ} \mathrm{C}$ for $30 \mathrm{~s}$. RT-PCR was performed using a Rotor- Gene Q thermocycler (Qiagen, Germany). A negative control containing RNAase/ DNAase-free water instead of cDNA was included in each run.

The expression of the target genes in both treated and untreated samples was analyzed and normalized against $r p o D$ expression. The level of gene expression in untreated and treated samples was calculated relative to the untreated PAO1.

\subsection{Molecular Docking}

CFP, CFPC, and CFPF were docked into the active site of $P$. aeruginosa ligand-binding domain at PDB ID: 1RO5 (Gould et al., 2004), PDB ID: 2UV0 (Bottomley et al., 2007) and PDB ID: 4JVD (Ilangovan et al., 2013) to evaluate their binding affinities, and to determine their inhibition activities and binding modes at the active site of LasI, LasR, and PqsR, respectively. The crystal structures of LasI, LasR, and PqsR were picked up from the protein data bank. All bound water ligands were removed from the protein. All components were constructed on ChemBioDraw using ChemBioOffice ultra v.14 software "ChemOffice, scientific personal productivity tools - PerkinElmer Informatics”. The energy was minimized by using MM2, Jop Type. Docking studies were performed using the Molsoft program, internal

TABLE 2 | Specific amplification primer sets for $P$. aeruginosa isolates.

\begin{tabular}{|c|c|c|c|c|c|}
\hline Gene type & Gene name & Type of primer & Primer Sequence & Melting temp. & Amplicon size (bp) \\
\hline \multirow[t]{2}{*}{ Reference gene. } & $r p o D$ & $\mathrm{Fw}$ & 5'-CGAACTGCTTGCCGACTT-3’ & $56^{\circ} \mathrm{C}$ & 131 \\
\hline & & Rev & 5-GCGAGAGCCTCAAGGATAC-3’ & & \\
\hline \multirow[t]{4}{*}{ QS genes. } & lasl & FW & 5'-CGCACATCTGGGAACTCA-3’ & $56^{\circ} \mathrm{C}$ & 176 \\
\hline & & Rev & 5`CGGCACGGATCATCATCT-3` & & \\
\hline & $r h l l$ & FW & 5-GTAGCGGGTITGCGGATG-3’ & $58^{\circ} \mathrm{C}$ & 101 \\
\hline & & Rev & 5'CGGCATCAGGTCTTCATCG-3` & & \\
\hline
\end{tabular}


coordinate mechanics (ICM) 3.4-8C was applied as reported by Abagyan et al. (1994) by converting PDB file 1RO5, 2UV0, and 4JVD into an ICM object. ICM aims to find the global minimum energy that describes the interaction between the ligand and the receptor. The modes of the interaction of the autoinducer molecule 3-oxo-C12-HSL within 2UV0 and 2-nonyl-4hydroxyquinoline (NHQ) within 4JVD were used as standard docked models.

\subsection{Statistical Analysis}

Each experiment was performed in triplicate. Mean and standard deviations of three independent measurements were calculated by Excel data package. Statistical analysis was performed using the GraphPad Instate software package (version 3.1) using $\mathrm{T}$ paired test for comparing the treated and untreated cultures. The results were assigned as significant when $p \leq 0.05$, and highly significant when $p \leq 0.01$ or $p \leq 0.001$.

\section{RESULTS}

\subsection{QSI of Metallic-Cefoperazone Derivatives Using C. violaceum ATCC 12472}

$\mathrm{CFP}, \mathrm{CFPF}$, and CFPC reduced violacein pigment production as indicated in table (1). The diameters of inhibition of violacein pigment were 25, 33, and $34 \mathrm{~mm}$ for CFP, CFPF, and CFPC, respectively (Table 1) (Supplementary Figure 1). However, CFP derivatization with $\mathrm{MnCl}_{2}, \mathrm{NiCl}_{2}$, and $\mathrm{CrCl}_{3}$ did not exhibit QSI activity (Supplementary Figure 1). Therefore, CFP and metalliccomplexes CFPF, and CFPC were selected to study their effects on virulence factors of $P$. aeruginosa clinical isolates and standard strains.

\subsection{Minimal Inhibitory Concentrations of CFP, CFPF, and CFPC}

Minimal inhibitory concentrations of CFP, CFPF, and CFPC against $P$. aeruginosa; Ps1, Ps2, Ps3, PAO1, PA14, and PAO-JP2 were evaluated. All tested strains were resistant to CFP with MIC $512 \mu \mathrm{g} / \mathrm{mL}$. CFPC showed a prominent antimicrobial activity with low MICs of 2, 4, 8, 8, 8, and $32 \mu \mathrm{g} / \mathrm{mL}$ against tested strains: Ps1, Ps2, Ps3, PAO1, PA14, and PAO-JP2, respectively. In addition, MICs of CFPF were $2,32,128,16,64$, and $16 \mu \mathrm{g} / \mathrm{mL}$ against $P$. aeruginosa strains: Ps1, Ps2, Ps3, PAO1, PA14, and PAO-JP2, respectively (Table 3).

\subsection{Effect of Sub-Inhibitory Concentrations of the Tested Compounds on the Growth of $P$. aeruginosa Isolates}

Viable count of $P$. aeruginosa was estimated after treating each isolate with 1/2 MIC of CFP, CFPC, and CFPF. Cultivation of $P$. aeruginosa with sub-MICs did not affect bacterial growth when compared to the control untreated cultures (Supplementary Table S1). For instance, the viable count of Ps1 isolate was 152,155 and $160 \times 10^{7} \mathrm{CFU} / \mathrm{mL}$ when treated with $1 / 2 \mathrm{MIC}$ of CFP, CFPC, and CFPF, respectively and the bacterial count of untreated Ps1 was $162 \times 10^{7} \mathrm{CFU} / \mathrm{mL}$. The counts of Ps2 treated with 1/2 MIC of CFP, CFPC, and CFPF were 140, 145 and $138 \times 10^{6} \mathrm{CFU} / \mathrm{mL}$, respectively while the count of untreated culture was $146 \times 10^{6} \mathrm{CFU} / \mathrm{mL}$. Also, the viable count of Ps 3 was 132,129 , and $134 \times 10^{7} \mathrm{CFU} / \mathrm{mL}$ upon addition of $1 / 2 \mathrm{MIC}$ of CFP, CFPC, and CFPF, respectively while the count of untreated culture was $136 \times 10^{7} \mathrm{CFU} / \mathrm{mL}$.

Furthermore, the viable count of PAO1 treated with $1 / 2 \mathrm{MIC}$ of CFP, CFPC, and CFPF were 166, 160, and $164 \times 10^{7} \mathrm{CFU} / \mathrm{mL}$, respectively and the viable count of untreated PAO1 was $168 \times$ $10^{7} \mathrm{CFU} / \mathrm{mL}$. Similarly, PA14 count was 175,168 , and $166 \times 10^{7}$ $\mathrm{CFU} / \mathrm{mL}$ when cultivated in 1/2 MIC of CFP, CFPC, and CFPF, respectively and the viable count of the untreated culture was $177 \times 10^{7} \mathrm{CFU} / \mathrm{mL}$. The viable count of PAO-JP2 culture was $181,184,180 \times 10^{7} \mathrm{CFU} / \mathrm{mL}$ when cultivated with $1 / 2 \mathrm{MIC}$ of CFP, CFPC, and CFPF, respectively and the viable count of the untreated culture was $187 \times 10^{7} \mathrm{CFU} / \mathrm{mL}$.

Additionally, the OD $600 \mathrm{~nm}$ of treated and untreated cultures was measured at different time intervals. There was no effect on the bacterial growth over time in cultures treated with 1/2 MIC of CFP, CFPC, and CFPF compared to untreated cultures (Supplementary Figure S2).

\subsection{Effect of Sub-MICs of the Tested Compounds on Virulence Factors}

The influences of sub-MICs; $1 / 2$ and $1 / 4$ of CFP, CFPC, and CFPF on the virulence factors; pyocyanin, protease, hemolysin, and biofilm of $P$. aeruginosa clinical isolates Ps1, Ps2 and Ps3, and standard strains PAO1, PA14, and PAO-JP2 were investigated and compared with untreated cultures at the same conditions.

TABLE 3 | MICs and sub-MICs; 1/2 and 1/4 of Cefoperazone (CFP), Cefoperazone-Cobalt complex (CFPC) and Cefoperazone-Iron complex (CFPF).

\begin{tabular}{|c|c|c|c|c|c|c|c|c|c|}
\hline & \multicolumn{3}{|c|}{ CFP } & \multicolumn{3}{|c|}{ CFPC } & \multicolumn{3}{|c|}{ CFPF } \\
\hline & $\begin{array}{c}\text { MIC } \mu \mathrm{g} / \\
\mathrm{mL}\end{array}$ & $\begin{array}{c}1 / 2 \mathrm{MIC} \mu \mathrm{g} / \\
\mathrm{mL}\end{array}$ & $\begin{array}{c}1 / 4 \mathrm{MIC} \mu \mathrm{g} / \\
\mathrm{mL}\end{array}$ & $\begin{array}{l}\text { MIC } \mu \mathrm{g} / \\
\mathrm{mL}\end{array}$ & $\begin{array}{c}\text { 1/2 MIC } \mu \mathrm{g} / \\
\mathrm{mL}\end{array}$ & $\begin{array}{c}\text { 1/4 MIC } \mu \mathrm{g} / \\
\mathrm{mL}\end{array}$ & $\begin{array}{c}\text { MIC } \mu \mathrm{g} / \\
\mathrm{mL}\end{array}$ & $\begin{array}{c}\text { 1/2 MIC } \mu \mathrm{g} / \\
\mathrm{mL}\end{array}$ & $\begin{array}{c}1 / 4 \mathrm{MIC} \mu \mathrm{g} / \\
\mathrm{mL}\end{array}$ \\
\hline P. aeruginosa Ps1 & 512 & 256 & 128 & 2 & 1 & 0.5 & 2 & 1 & 0.5 \\
\hline P. aeruginosa Ps2 & 512 & 256 & 128 & 4 & 2 & 1 & 32 & 16 & 8 \\
\hline P. aeruginosa Ps3 & 512 & 256 & 128 & 8 & 4 & 2 & 128 & 64 & 32 \\
\hline P. aeruginosa PAO1 & 512 & 256 & 128 & 8 & 4 & 2 & 16 & 8 & 4 \\
\hline P. aeruginosa PA14 & 512 & 256 & 128 & 8 & 4 & 2 & 64 & 32 & 16 \\
\hline P. aeruginosa PAO- & 512 & 256 & 128 & 32 & 16 & 8 & 16 & 8 & 4 \\
\hline
\end{tabular}

JP2 


\subsubsection{Pyocyanin Assay}

CFP at $1 / 2$ MIC $(256 \mu \mathrm{g} / \mathrm{mL})$ significantly $(p<0.001)$ inhibited pyocyanin production in $P$. aeruginosa strains; Ps1, Ps2, Ps3, PAO1 and PA14 by $79.0 \%, 88.6 \%, 99.4 \%, 97.5 \%$ and $98.7 \%$, respectively. Also, 1/4 MIC of CFP reduced pyocyanin by $78.9 \%$, 87.2\%, 99.3\%, 97.5\% and 98.7\% in Ps1, Ps2, Ps3, PAO1 and PA14, respectively $(p<0.001)$. Interestingly, CFPC at a significantly low concentrations (1-16 $\mu \mathrm{g} / \mathrm{mL})$ reduced pyocyanin production by $79.4 \%, 68.5 \%, 56.6 \%, 24.4 \%$ and $25.5 \%$ in Ps1, Ps2, Ps3, PAO1 and PA14, respectively. Moreover, $1 / 4 \operatorname{MIC}(0.5,1,2,2$ and $2 \mu \mathrm{g} / \mathrm{mL})$ of CFPC significantly lowered pyocyanin levels by $71.8 \%, 61.4 \%, 55.1 \%$, and $25 \%$ in Ps1, Ps2, Ps3, and PA14, respectively. On the same instance, $1 / 2$ MIC of CFPF significantly inhibited pyocyanin production by $80.4 \%, 46.7 \%, 45.2 \%, 41.6 \%, 39.7 \%$ and $40.1 \%$ in Ps1, Ps2, Ps3, PAO1 and PA14 cultures, respectively. Furthermore, 1/4 MIC of CFPF $(0.5,8,32,4$ and $16 \mu \mathrm{g} / \mathrm{mL})$ significantly reduced pyocyanin by $62.4 \%, 50.0 \%, 34.9 \%, 31.7 \%$ and $28.8 \%$ in Ps1, Ps2, Ps3, PAO1 and PA14, respectively $(p<0.001$ and $p<0.01)$ (Figure 1).

\subsubsection{Total Protease Assay}

CFP at $1 / 2$ MIC reduced protease production by $34.5 \%(p<0.01)$, 7.6\%, 2.4\%, 6.6\% and 23.5\% $(p<0.05)$ in Ps1, Ps2, Ps3, PAO1 and
PA14 strains, respectively. Pseudomonas isolates Ps1, Ps2, Ps3, PAO1 and PA14 supplied with 1/4 MIC exhibited decrease in protease levels by $33.3 \%(p<0.01), 8.9 \%, 3.5 \%, 7.7 \%$ and $24.5 \%$ $(p<0.05)$, respectively. CFPC at $1 / 2 \mathrm{MIC}$ inhibited proteolytic activity by $20.7 \%, 26.6 \%, 28.2 \%, 14.3 \%$ and $16.3 \%$ in Ps1, Ps2, Ps3, PAO1 and PA14, respectively. CFPC at 1/4 MIC reduced protease by $10.3 \%, 6.3 \%, 18.8 \%, 18.7 \%$ and $9.2 \%$ in Ps1, Ps2, Ps3, PAO1 and PA14, respectively. Sub-MICs of CFPF at $1 / 2$ MIC lowered protease activity by $24.1 \%, 28 \%, 37.6 \%, 27.5 \%$ and $33.7 \%$ in Ps1, Ps2, Ps3, PAO1 and PA14, respectively. CFPF at $1 / 4 \mathrm{MIC}$ reduced protease by $13.8 \%, 20.7 \%, 27.1 \%, 30.8 \%$ and $36.7 \%$ in Ps1, Ps2, Ps3, PAO1 and PA14, respectively. PAO-JP2 showed the least protease activity of 0.036 at OD $600 \mathrm{~nm}$ (Figure 2).

\subsubsection{Hemolysin Activity}

Sub-MICs of CFP significantly inhibited hemolysin production in Ps1, Ps2, Ps3, PAO1 and PA14 by 38\%, 42.2\%, 48.4\%, 50\% and $53.6 \%$, respectively when supplied with $1 / 2 \mathrm{MIC}(256 \mu \mathrm{g} / \mathrm{mL})$. Adding CFP at $1 / 4$ MIC $(128 \mu \mathrm{g} / \mathrm{mL})$ significantly $(p<0.01)$ reduced hemolysin by $33.8 \%, 41 \%, 43.8 \%, 49.8 \%$ and $50.5 \%$ in Ps1, Ps2, Ps3, PAO1 and PA14, respectively. CFPC at 1/2 MIC showed significant reduction $(p<0.01)$ in hemolysin activity by $33.8 \%, 44.6 \%, 76.6 \%, 46.9 \%$ and $48.5 \%$ in Ps1, Ps2, Ps3, PAO1 and PA14, respectively. Also, $1 / 4$ MIC significantly $(p<0.01)$

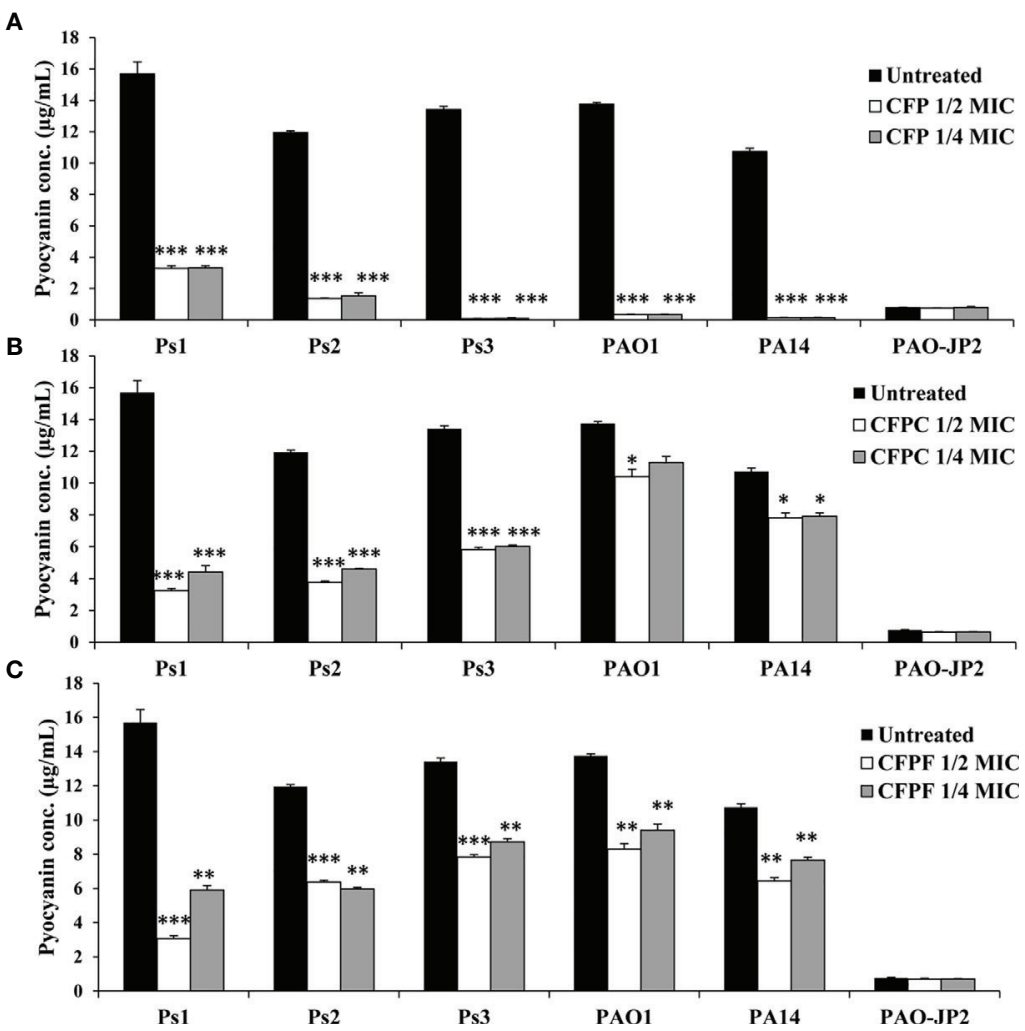

FIGURE 1 | Pyocyanin production; Effect of sub-MICs; 1/4 and 1/2 of Cefoperazone (CFP) (A) Cefoperazone-Cobalt complex (CFPC) (B) and Cefoperazone-Iron complex (CFPF) (C) on pyocyanin production in P. aeruginosa strains, Ps1, Ps2, Ps3, PAO1, PA14 and PAO-JP2 (negative control) compared to the untreated cultures. Error bars represent S.D. $(\mathrm{n}=3),{ }^{\star} p \leq 0.05,{ }^{\star \star} p \leq 0.01$, and ${ }^{\star * *} p \leq 0.001$. 


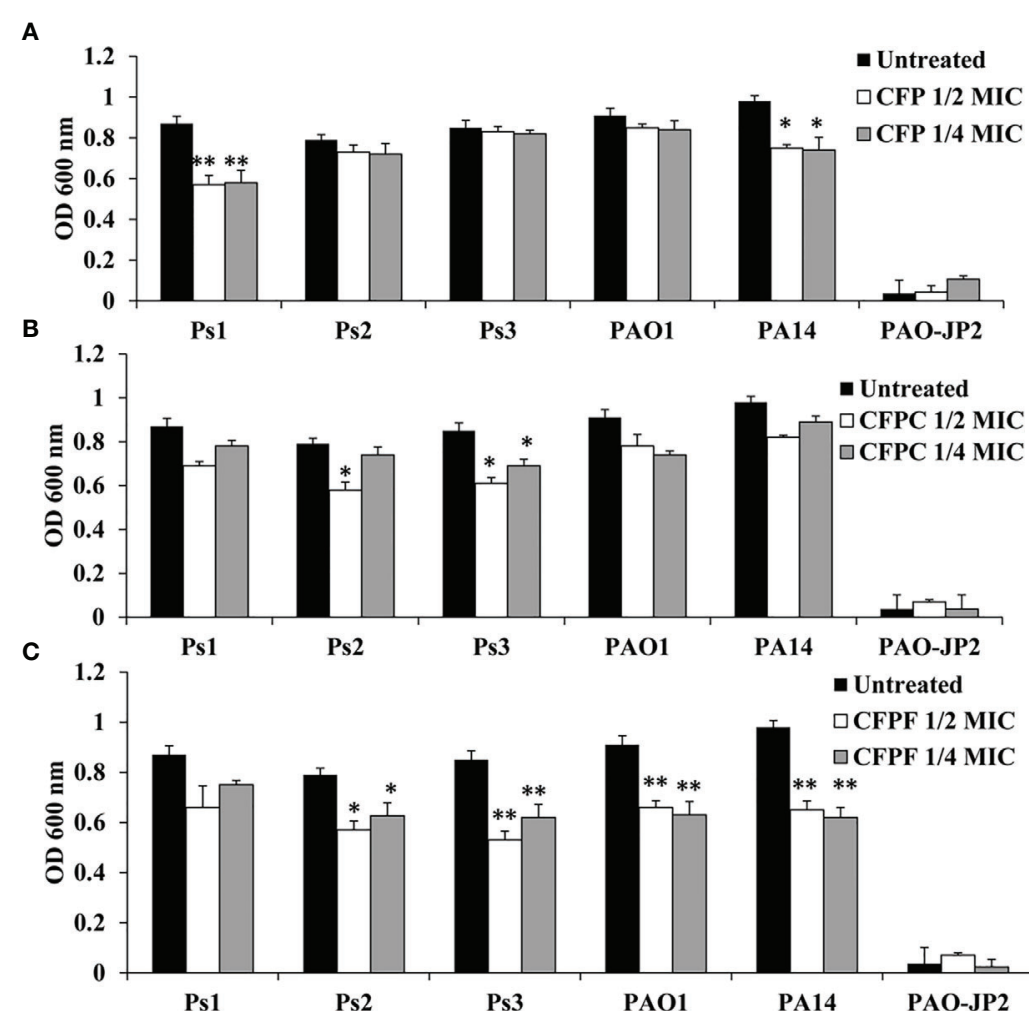

FIGURE 2 | Protease production; Effect of sub-MICs; 1/4 and 1/2 of Cefoperazone (CFP) (A) Cefoperazone-Cobalt complex (CFPC) (B) and Cefoperazone-Iron complex (CFPF) (C) on total protease production in P. aeruginosa strains, Ps1, Ps2, Ps3, PAO1, PA14 and PAO-JP2 (negative control) compared to the untreated cultures. Error bars represent S.D. $(\mathrm{n}=3),{ }^{\star} p \leq 0.05$, and ${ }^{* *} p \leq 0.01$.

inhibited hemolysin by $32.4 \%, 43.4 \%, 68.8 \%, 36.7 \%$ and $37.1 \%$ in Ps1, Ps2, Ps3, PAO1 and PA14, respectively. Similarly, 1/2 MIC of CFPF significantly reduced hemolysin by $56.9 \%, 60.4 \%, 73.4 \%$, 79.6\% and 50.5\% in Ps1, Ps2, Ps3, PAO1 and PA14, respectively. Also, 1/4 MIC of CFPF reduced hemolysin by $39.2 \%, 38.6 \%$, $73.4 \%, 79.6 \%$ and $50.5 \%$ in Ps1, Ps2, Ps3, PAO1 and PA14, respectively $(p<0.01)$. The negative control PAO-JP2 exhibited the least hemolysin activity as 0.11 at OD $540 \mathrm{~nm}$ (Figure 3).

\subsubsection{Effect on Biofilm Formation}

Using $1 / 2$ MIC of CFP significantly $(P<0.01)$ inhibited biofilm formation by Ps1, Ps2, Ps3, PAO1 and PA14 by $61.1 \%, 84 \%$, $36.1 \%, 62 \%$ and $85.6 \%$, respectively. Treatment with $1 / 4 \mathrm{MIC}$ of CFP $(128 \mu \mathrm{g} / \mathrm{mL})$ eliminated biofilm by $56.9 \%, 81.3 \%, 37.6 \%$, $54.8 \%$ and $82.5 \%$, respectively. Surprisingly, CFPC at $1 / 2 \mathrm{MIC}$ significantly reduced biofilm formation by $30.6 \%, 34.8 \%, 21.2 \%$, $35.4 \%$ and $62 \%$ in Ps1, Ps2, Ps3, PAO1 and PA14, respectively. At the same instance, $1 / 4$ MIC of CFPC significantly reduced biofilm by $29.9 \%, 34 \%, 19 \%, 38.9 \%$ and $51.8 \%$, respectively. Furthermore, CFPF significantly diminished biofilm formation by Ps1, Ps2, Ps3, PAO1 and PA14 when supplied with 1/2 MIC by $34 \%, 42 \%, 22 \%, 46.9 \%$ and $58 \%$, respectively. Using $1 / 4 \mathrm{MIC}$ of CFPF reduced biofilm by $33.3 \%, 33 \%, 9.8 \%, 29.2 \%$ and $52.1 \%$ in $P$. aeruginosa strains; Ps1, Ps2, Ps3, PAO1 and PA14, respectively (Figure 4).

\subsection{Expression of QS-Regulated Genes}

To get insights into the molecular mechanism of CFP, CFPC and CFPF in reducing QS of $P$. aeruginosa. RT- PCR was used to assess relative expression levels of lasI, and rhlI genes. CFP, CFPC and CFPF was found to significantly $(p<0.001)$ reduce the transcription of lasI by $77.25 \%, 94 \%$ and $88 \%$, respectively in $\mathrm{PAO} 1$ compared to the untreated samples (Figure 5A).

Also, significant inhibition in rhlI gene expression in $P$. aeruginosa $\mathrm{PAO} 1$ was elucidated when subjected to CFP, CFPF and CFPC at sub-MIC. The relative expression data showed a significant reduction in rhlII gene expression by $44 \%$ in CFP treated cells $(p<0.01), 41 \%$ reduction in CFPF treated culture $(p<0.01)$ and $80 \%$ reduction in CFPC treated cells $(p<0.001)$ when compared to the untreated cells (Figure 5B).

\subsection{Binding Affinity Analysis for LasR and Ligands}

The ICM score and hydrogen bonds between compounds and the surrounding amino acids were used to predict the binding modes, their binding affinities, and the orientation of these compounds at the active site of LasI, LasR, and PqsR. The scoring functions of the compounds were calculated from minimized ligand-receptor complexes. CFP, CFPC and CFPF were docked at the binding site of LasI. CFP showed high ICM score of -123.78 and formed seventeen hydrogen bonds with 


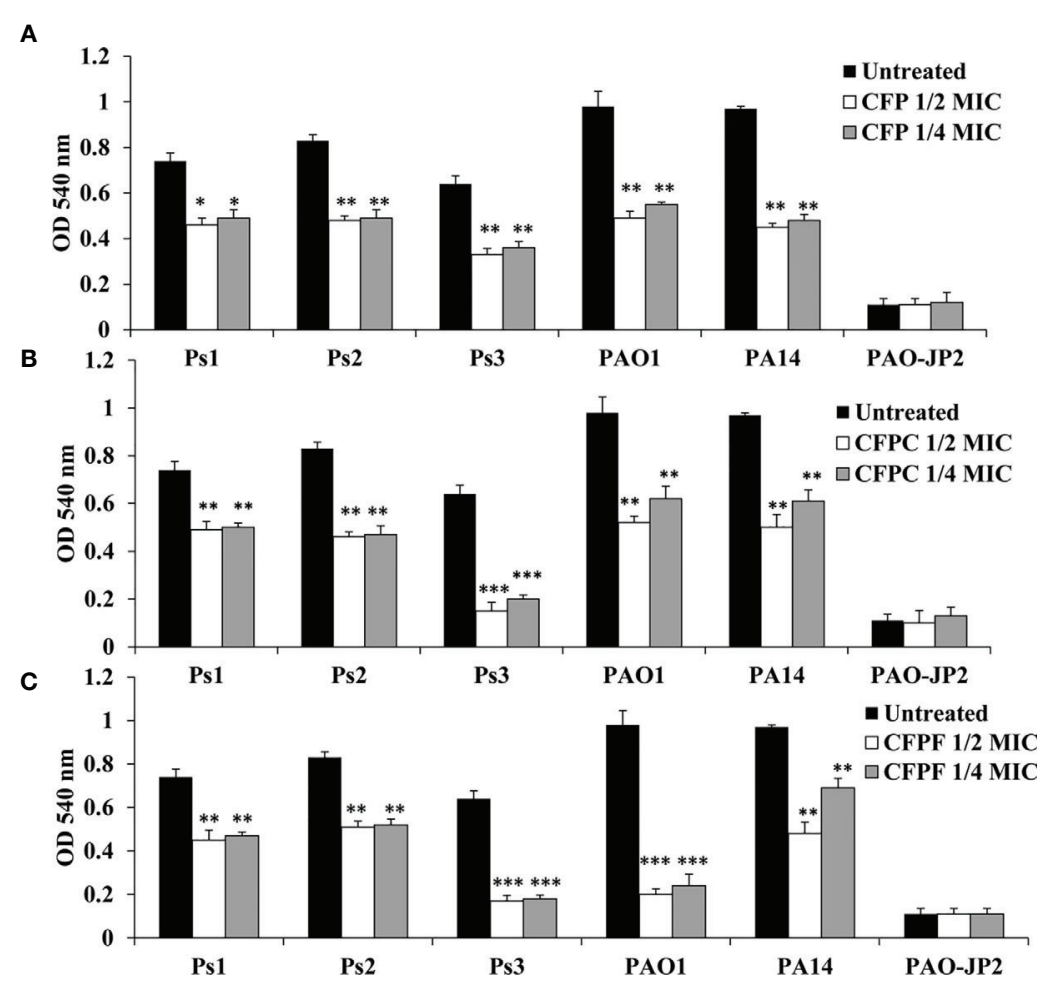

FIGURE 3 | Hemolysin activity; Effect of sub-MICs; 1/4 and 1/2 of Cefoperazone (CFP) (A) Cefoperazone-Cobalt complex (CFPC) (B) and Cefoperazone-Iron complex (CFPF) (C) on hemolysin activity measured at OD $540 \mathrm{~nm}$ in P. aeruginosa strains, Ps1, Ps2, Ps3, PAO1, PA14 and PAO-JP2 (negative control) compared to the untreated cultures. Error bars represent S.D. $(n=3),{ }^{\star} p \leq 0.05$, ${ }^{\star *} p \leq 0.01$, and ${ }^{\star \star *} p \leq 0.001$.

Arg30, Arg104, Ile107, Thr144, Lys150, Arg172, and Glu171. Both derivatives; CFPC and CFPF gave higher ICM scores of -132.85 and -191.69 , respectively (Table 4). CFPC binds with the receptor by ten hydrogen bonds with Lys167, Arg172, Ile170, and Glu171. While CFPF form nine hydrogen bonds with Arg30, Arg104, Thr144, Thr145, Phe105, and Thr121 (Figure 6, Supplementary Table S2).

To validate the docking protocol, the autoinducer 3-oxo-C12HSL was docked in its binding site LasR, which revealed ICM score of -107.47 and form three hydrogen bonds with Trp60, Asp73, and Ser129. CFP showed ICM score of -112.96 and formed ten hydrogen bonds, one hydrogen bond with each of Tyr47, Tyr64, Thr75: two hydrogen bonds with each of Ala50 and Gly126 and three hydrogen bonds with Arg61. CFPC and CFPF gave ICM scores of -136.44 and -179.52 , respectively which is higher than the autoinducer's ICM score (-107.47) (Table 4). CFPC bound with the receptor by twenty-six hydrogen bonds with amino acids Tyr47, Ala50, Gly54, Trp60, Arg61, Tyr64, Asp65, Asp73, Pro74, Thr75, Val76, Ser77, Ile86, Phe87, and Gln98. While CFPF form fourteen hydrogen bonds with Tyr47, Asn49, Ala50, Gly54, Trp60, Arg61, Tyr64, Asp73, Thr75 (Figure 7, Supplementary Table S3).

Also, NHQ was docked into its binding site PqsR, it revealed ICM score of -57.78 and formed three hydrogen bonds with Gln194, Ile236, and Leu208. CFP, CFPC and CFPF showed ICM score of $-105.37,-130.05$ and -161.85 , respectively (Table 4).
CFP formed fourteen hydrogen bonds with Arg126, Ser128, Asp131, Thr135, Arg200, and Asn220. CFPC bound to the receptor by seventeen hydrogen bonds with Lys167, Asp264, Thr265, and Lys266. While CFPF formed twenty-eight hydrogen bonds with Ser128, Ala130, Asp131, Ser132, Leu133, Ala134, Thr135, Gly198, Arg200, Ser201, Gln203, Ser205, and Glu219 (Figure 8, Supplementary Table S4).

Molecular docking of CFP, CFPC and CFPF with LasI, LasR, and PqsR of $P$. aeruginosa are indicated in the Supplementary Tables S2-S4.

\section{DISCUSSION}

$P$. aeruginosa is a common opportunistic organism with a remarked ability for survival in various environmental conditions. QS is the key element that regulates gene expression and virulence behaviors such as proteases, hemolysin, pyocyanin pigment, and biofilm formation (Schuster and Greenberg, 2006; Thornton et al., 2020). Recently, treatment of $P$. aeruginosa infection represents a great challenge due to the high incidence of drug resistance. So, the development of a new therapeutic approach is imperative, one of these approaches is targeting QS cascade by inhibitors to control Pseudomonas virulence factors. Hence, bacterial infection can be easily eliminated by the immune system 


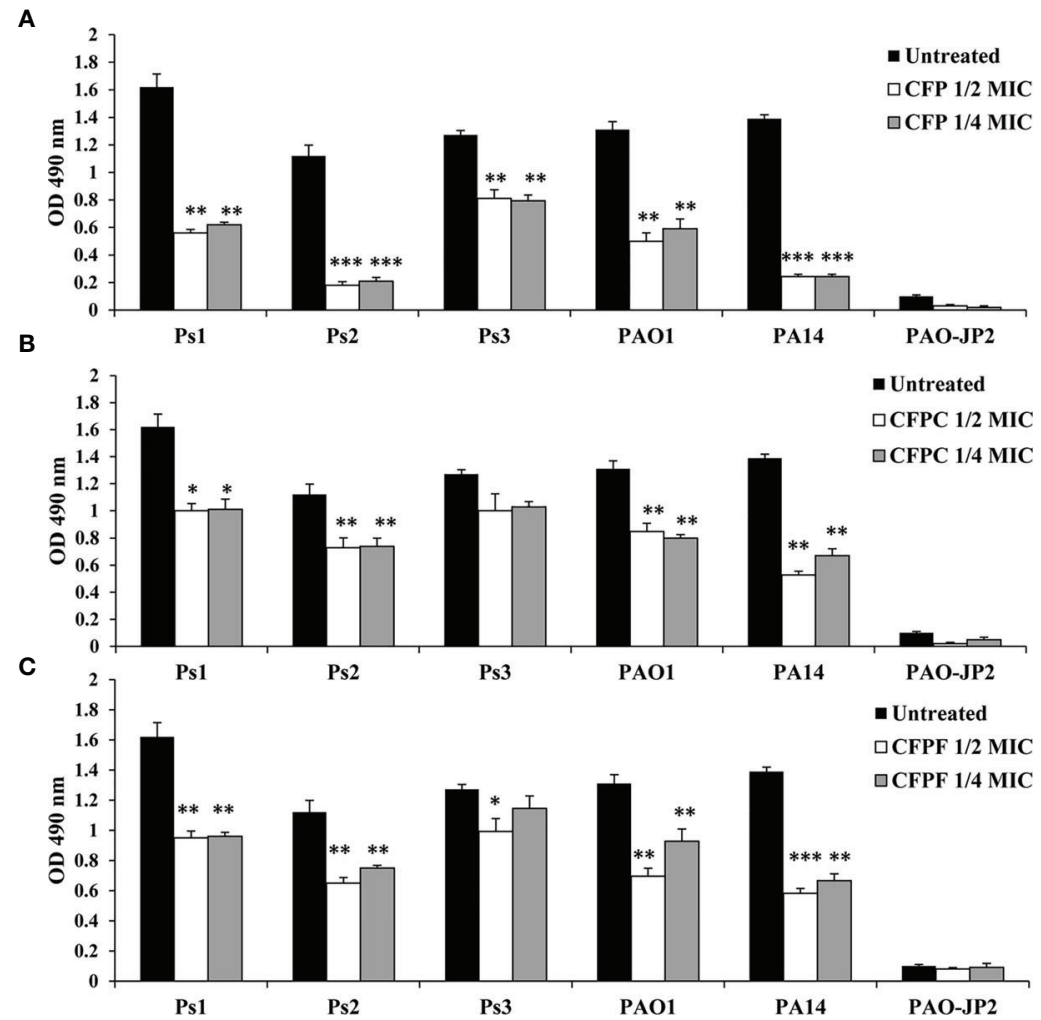

FIGURE 4 | Biofilm formation; Effect of sub-MICs; 1/4 and 1/2 of Cefoperazone (CFP) (A), Cefoperazone-Cobalt complex (CFPC) (B) and Cefoperazone-Iron complex (CFPF) (C) on biofilm formation in P. aeruginosa strains, Ps1, Ps2, Ps3, PAO1, PA14 and PAO-JP2 (negative control) compared to the untreated cultures. Error bars represent S.D. $(n=3),{ }^{\star} p \leq 0.05,{ }^{\star *} p \leq 0.01$, and ${ }^{\star \star *} p \leq 0.001$.

A

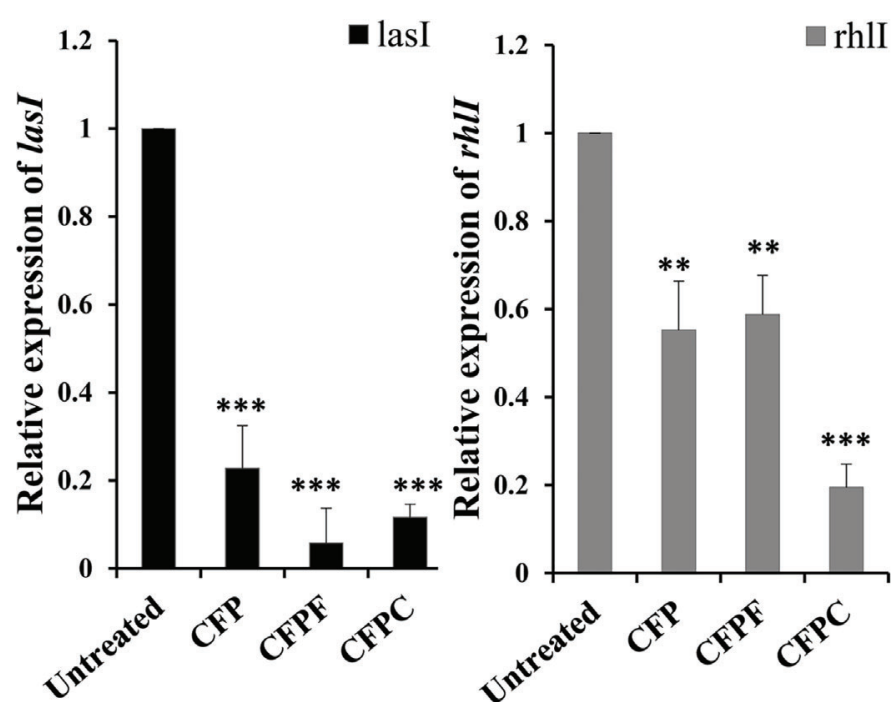

FIGURE 5 | Cefoperazone (CFP), Cefoperazone-Cobalt complex (CFPC) and Cefoperazone-Iron complex (CFPF) at 1/2 MIC inhibited QS regulatory genes (A) las/ and $\mathbf{( B )} r h l l$ of $P$. aeruginosa PAO1 compared to the expression of genes in untreated PAO1. Error bars represent $\mathrm{S} . \mathrm{D}$. $(\mathrm{n}=3)^{* \star} p \leq 0.01$ and ${ }^{\star \star \star} p \leq 0.001$. 
TABLE 4 | Internal coordinate mechanics scores, no. of $\mathrm{H}$-bonds and amino acid residues involved in the interaction between LasR, Lasl and PqsR binding site with tested compounds and the autoinducer molecule of the receptor.

\begin{tabular}{|c|c|c|c|c|}
\hline Receptor & Compound & ICM Score & $\begin{array}{c}\text { No. of } \\
\text { H-Bonds }\end{array}$ & Amino acid residues involved \\
\hline \multirow[t]{4}{*}{ Lasl } & Sulphate & -44.03 & 12 & Arg30. \\
\hline & CFP & -123.78 & 17 & Arg30, Arg104, lle107, Thr144, Lys150, Arg172, Glu171. \\
\hline & CFPC & -132.85 & 10 & Lys167, Arg172, Ile170, Glu171. \\
\hline & CFPF & -191.69 & 9 & Arg30, Arg104, Thr144, Thr145, Phe105, Thr121. \\
\hline \multirow[t]{4}{*}{ LasR } & 3-oxo-C12-HSL & -107.47 & 3 & Trp60, Asp73, Ser129. \\
\hline & CFP & -112.96 & 10 & Tyr47, Ala50, Arg61, Tyr64, Thr75, Gly126. \\
\hline & CFPC & -136.44 & 26 & Tyr47, Ala50, Gly54, Trp60, Arg61, Tyr64, Asp65, Asp73, Pro74, Thr75, Val76, Ser77, Ile86, Phe87, Gln98 \\
\hline & CFPF & -179.52 & 14 & Tyr47, Asn49, Ala50, Gly54, Trp60, Arg61, Tyr64, Asp73, Thr75. \\
\hline \multirow[t]{4}{*}{ PqsR } & NHQ & -57.78 & 3 & Gln194, Ile236, Leu208. \\
\hline & CFP & -105.37 & 14 & Arg126, Ser128, Asp131, Thr135, Arg200, Asn220. \\
\hline & CFPC & -130.05 & 17 & Lys167, Asp264, Thr265, Lys266. \\
\hline & CFPF & -161.85 & 28 & Ser128, Ala130, Asp131, Ser132, Leu133, Ala134, Thr135, Gly198, Arg200, Ser201, Gln203, Ser205, Glu219. \\
\hline
\end{tabular}

without the concern of microbial resistance (Hentzer and Givskov, 2003).

According to our knowledge, less is known about QSI activity of CFP, and no data is available about the antiquorum sensing effects of CFPC and CFPF. In this study, we evaluated the antiquorum sensing activities of $\mathrm{CFP}$ using $C$. violaceum ATCC 12472 reporter strain. C. violaceum ATCC 12472 produces several $\mathrm{N}$-acyl-L-homoserine lactones (AHLs); $\mathrm{N}$ nonanoyl-homoserine lactone, $\mathrm{N}$-undecanoyl-homoserine lactone, $\mathrm{N}$-(3-oxodecanoyl)-homoserine lactone (3-oxo-C10-
HSL), N-(3-hydroxydecanoyl)-L-homoserine lactone, $\mathrm{N}$-(3hydroxyundecanoyl)-homoserine lactone, and 3-oxo-C12-HSL (Mion et al., 2021). N-(3-hydroxydecanoyl)-L-homoserine lactone is the main inducer that controls violacein production via quorum sensing. Other signaling molecules such as 3-oxoC10-HSL and 3-oxo-C12-HSL can enhance the production of violacein in C. violaceum ATCC 12472 (Morohoshi et al., 2008). In this study, CFP, and its metallic derivatives CFPC and CFPF inhibited violacein pigment (Table 1) without any effect on microbial growth. Similarly, the QSI activity of flavonoid from<smiles>C=CC(C)(C)C(C)(C)C(C)=C(C)C(C)C</smiles>

C

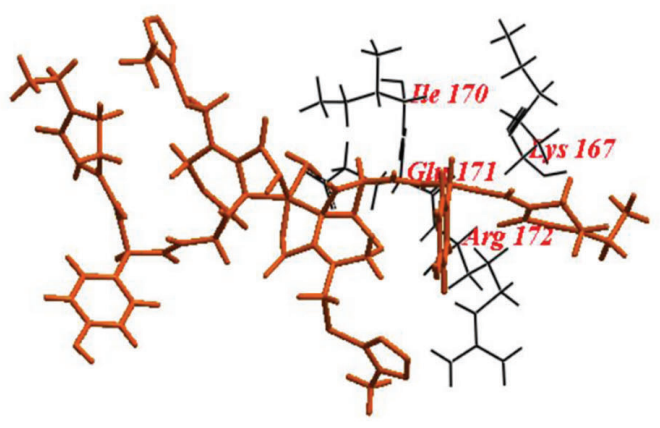

D
B
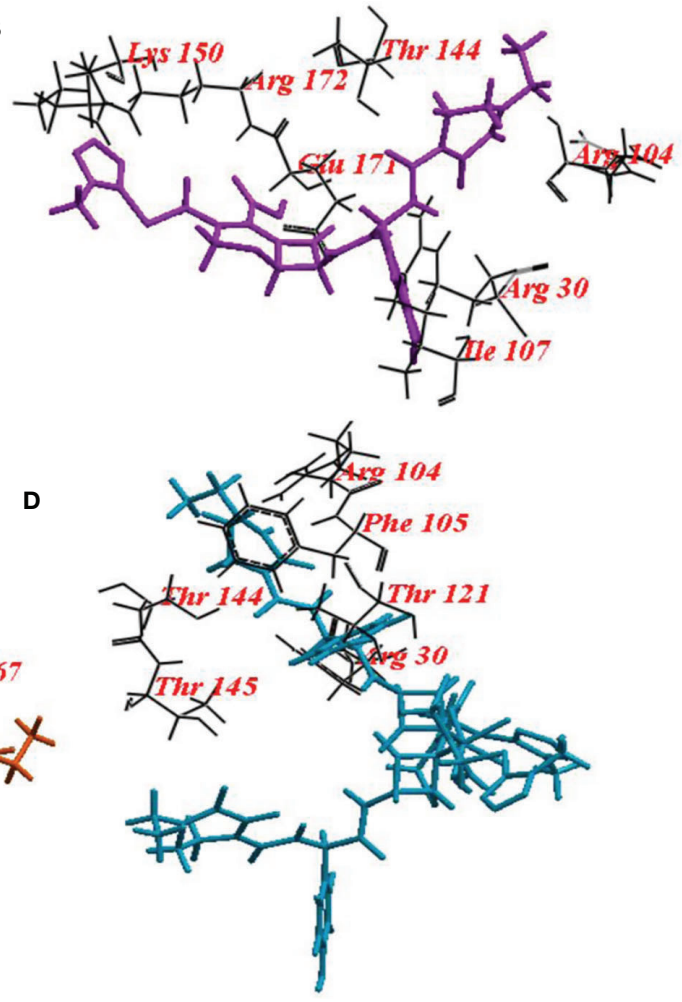

FIGURE 6 | Binding mode of (A) redocked sulphate ligand; (B) Cefoperazone (CFP); (C), Cefoperazone-Cobalt complex (CFPC) and (D) Cefoperazone-Iron complex (CFPF) into the Crystal structure $P$. aeruginosa acyl-homoserinelactone synthase Lasl. 


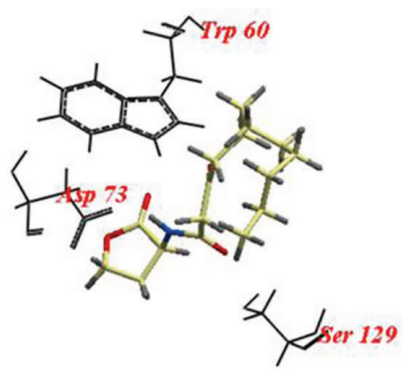

c

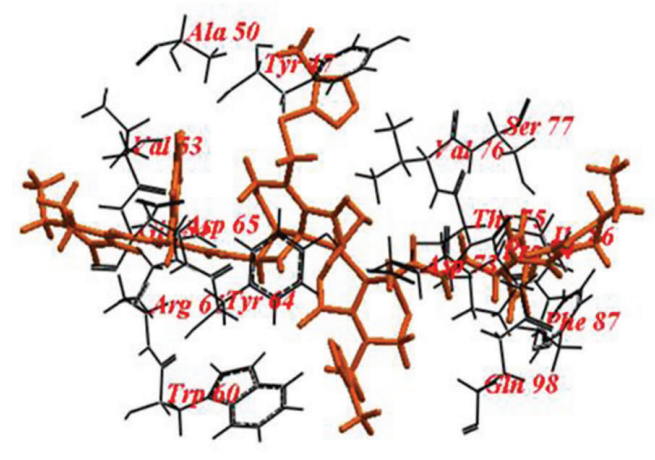

B

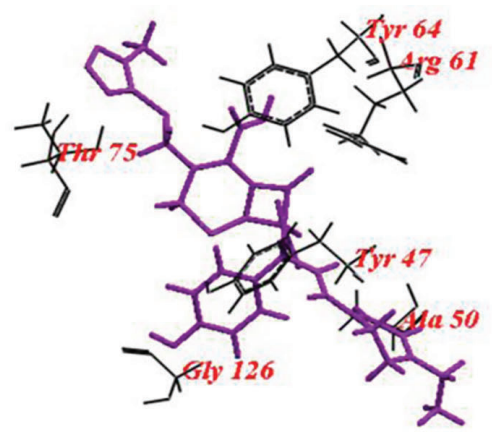

D

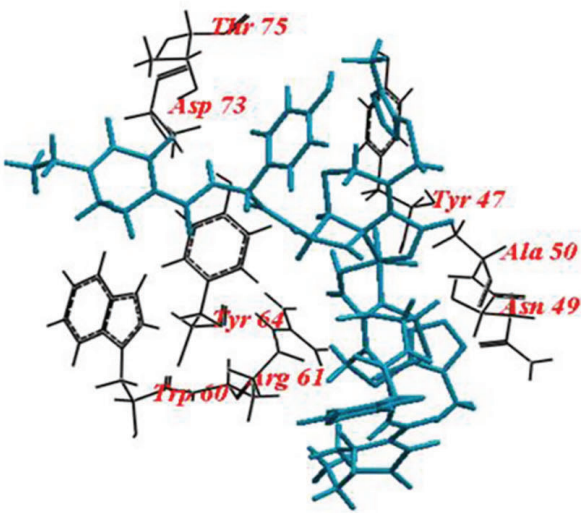

FIGURE 7 | Binding mode of (A) 3-oxo-C12-HSL cefoperazone; (B) Cefoperazone (CFP); (C), Cefoperazone-Cobalt complex (CFPC) and (D) Cefoperazone-Iron complex (CFPF) and redocked into the active site of $P$. aeruginosa ligand-binding domain.

Cassia alata (Rekha et al., 2017), quercetin and quercetin-3-Oarabinoside from Psidium guajava (Vasavi et al., 2014), curcumin, apigenin and luteolin (Burcu Bali et al., 2019), and enantiopure alkylglycerols (Chaverra Daza et al., 2021) inhibited violacein pigment production of C. violaceum ATCC 12472 and eliminated quorum sensing associated virulence factors of $P$. aeruginosa. QSI activity of antimicrobial agents has been previously reported including azithromycin (Bala et al., 2011), ciprofloxacin, tobramycin, and ceftazidime (Garske et al., 2004; Skindersoe et al., 2008).

Interestingly, CFPC and CFPF reduced QS-related virulence factors of $P$. aeruginosa without any effect on cell viability. For instance, $P$. aeruginosa produces green pyocyanin pigment after 24-48 hours of growth under the control of PQS and rhlI/R signaling systems (Gupta et al., 2011). CFP reduced pyocyanin levels in all $P$. aeruginosa strains both clinical isolates and standard strains after treatment by $1 / 2$ and $1 / 4$ MICs (128-256 $\mu \mathrm{g} / \mathrm{mL})$. At 2-8 folds low concentrations, CFPC $(0.5-16 \mu \mathrm{g} / \mathrm{mL})$ and CFPF $(0.5-64 \mu \mathrm{g} / \mathrm{mL})$ significantly reduced pyocyanin production to the level of PAO-JP2 (negative control) (Figure 1). Also, CFP, CFPC, and CFPF inhibited las-regulated virulence genes such as protease and hemolysin (Figures 2, 3). These results are consistent with reports that $\beta$-lactams antibiotics such as cefepime, ceftazidime, and imipenem inhibit pyocyanin, protease, and hemolysin production in $P$. aeruginosa
PAO1 without affection microbial viability (El-Mowafy et al., 2017; Aleanizy et al., 2021). Also, previous findings showed that metformin (Abbas et al., 2017) and zinc oxide nanoparticles (Saleh et al., 2019) inhibited hemolytic activity of $P$. aeruginosa.

Additionally, $P$. aeruginosa has a pronounced ability for biofilm assembly. Pseudomonas biofilms are resistant to most of the conventional antibiotic regimens. Inhibition of biofilm formation makes bacterium more susceptible to immune system and phagocytosis by neutrophils (Hentzer et al., 2002; Hentzer and Givskov, 2003). CFP, CFPC, and CFPF at $1 / 2$ and $1 / 4$ MICs showed a significant reduction in biofilm formation (Figure 4). Quorum sensing regulates the production of several extracellular virulence factors and promotes biofilm maturation, meaning that it has a key role in the pathogenesis of $P$. aeruginosa (Wagner et al., 2003). Azithromycin (Swatton et al., 2016), imipenem (ElMowafy et al., 2017), piperacillin/tazobactam (Aleanizy et al., 2021) inhibit biofilm formation via interruption with the QS cascade. In addition, QSI activity of garlic renders P. aeruginosa biofilms sensitive to tobramycin and phagocytosis (Bjarnsholt et al., 2005).

On the molecular level, we tested the activity of CFP, CFPC, and CFPF on the expression of QS genes lasI, and rhlI. Data revealed that CFP, CFPC, and CFPF at 1/2 MIC exhibited significant reduction in the relative expression of lasI by $77.25 \%, 94 \%$ and $88 \%$, respectively (Figure 5). The decrease in 
A

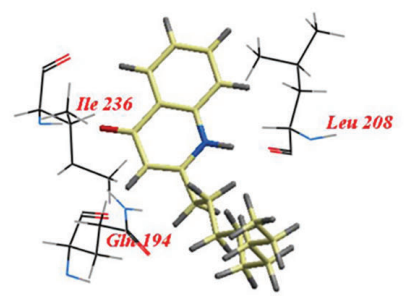

C

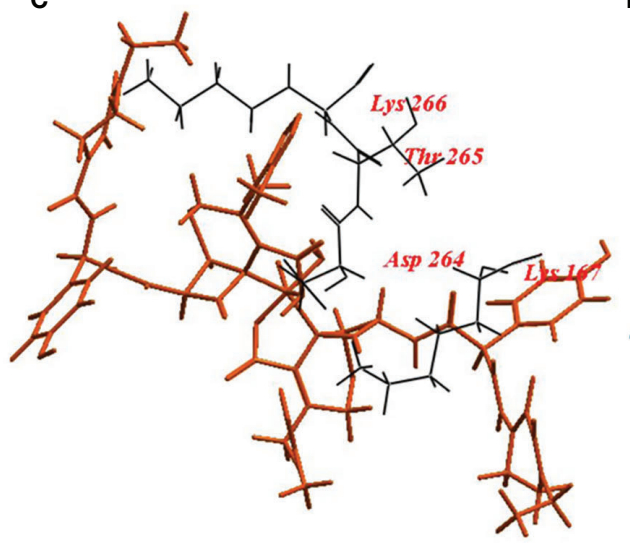

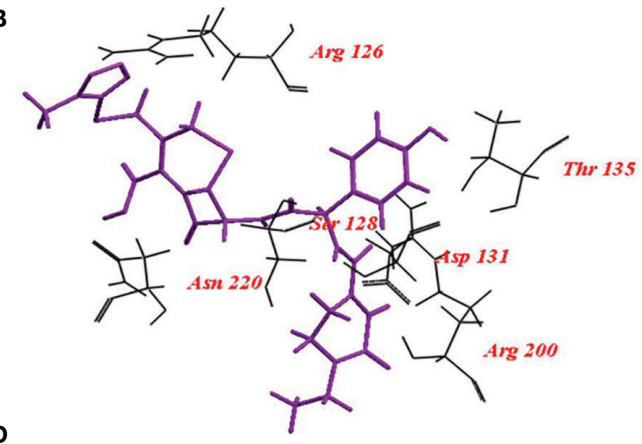

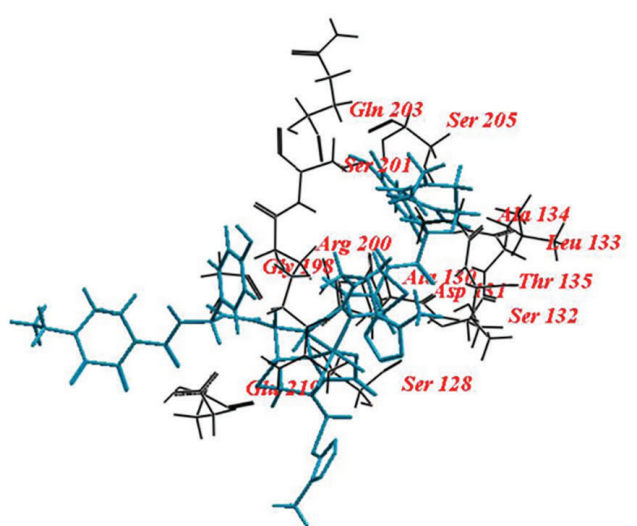

FIGURE 8 | Binding mode of (A) 2-nonyl-4-hydroxyquinoline (NHQ) redocked ligand; (B) Cefoperazone (CFP); (C), Cefoperazone-Cobalt complex (CFPC) and (D) Cefoperazone-Iron complex (CFPF) into the Crystal structure of PqsR coinducer binding domain of $P$. aeruginosa.

the expression level of lasI was consistent with reduction in all virulence factors controlled by lasI/R. The interaction of LasR with 3-oxo-C12-HSL, induces las system and triggers the production of virulence factors such as elastase, alkaline protease, hydrogen-cyanide synthase, exotoxin A, and secretion apparatus (Pessi et al., 2001). Previous researches elucidated that inhibition of lasI/R QS circuit is associated with reduction of $P$. aeruginosa virulence traits including elastase, total protease, hemolysin, and biofilm (Tateda et al., 2001; Gupta et al., 2011).

Also, the relative expression of rhlI was significantly reduced by CFP, CFPC, and CFPF (Figure 5). The lasI/R and $r h l I / r h l R$ circuits are interconnected and suppression of lasI is associated with subsequent inhibition of rhlI gene expression (Pesci et al., 1997). The $r h l$ system is also necessary for optimal production of lasB elastase, lasA protease, pyocyanin (Latifi et al., 1996). Furthermore, the rhl system is involved in the regulation of hcnABC, and alkaline protease (Pearson et al., 1997; Pessi et al., 2001).

For a deep understanding of the CFP, CFPC, and CFPF activities, docking of these compounds was performed at the active site of LasI, LasR, and PqsR. Docking study of LasI revealed higher ICM scores exhibited by CFP, CFPC and CFPF than that of the natural legend (Table 4). CFP bound the receptor with Arg30, Arg104, Ile107, Thr144, Lys150, Arg172, and Glu171. CFPC bind with Lys167, Arg172, Ile170, and Glu171. While CFPF bound with Arg30, Arg104, Thr144,
Thr145, Phe105, and Thr121 (Figure 6). Several of these residues, including Met79, Phe105, Thr142 and Thr144, are well conserved among AHL-synthases. The basic residues, Lys150, Arg154, Arg161, His165, Lys167, and Arg172 form a highly positive charged patch on the LasI surface (Gould et al., 2004). Likewise, carvacrol interacts with LasI synthase Arg104 residue through Van der Waals forces and occupies the LasR binding site pocket at Asp73 residue, with subsequent elimination of the expression of virulence factors in $P$. aeruginosa (Tapia-Rodriguez et al., 2019).

The amino acid residues in the active site of LasR are Ala50, Tyr56, Trp60, Tyr64, Asp73, Thr75, Cys79, Gly126, Trp88, Tyr93, Ala105, Leu110, and Ser12 as indicated in the pdbsumb (PDB ID: 2UV0). The hydrogen bonds between the polar groups of the acyl-homoserine lactone and residues Tyr56, Trp60, Asp73, and Ser129 of LasR are pivotal for the correct folding of LuxR protein (Monterrosa et al., 2019; Prateeksha et al., 2019; Hnamte et al., 2020). Ahmed and coauthors elucidate the binding of 3-oxo-C12-HSL with LasR via hydrogen bonds with Tyr56, Trp60, and Asp73 (Ahmed et al., 2020). In our study, 10 hydrogen bonds were formed between CFP and LasR receptor at Tyr47, Tyr64, Thr75, Ala50, Gly126, and Arg61 residues. The binding energy of CFP (-122.96), CFPC (-141.38) and CFPF $(-179.52)$ with LasR was higher than that of 3-oxo-C12-HSL (-107.47) (Figure 5 and Table 4). Therefore, the high binding affinity of CFPC and CFPF with LasR receptors could eliminate 
the binding of natural ligand 3-oxo-C12-HSL with LasR receptors (Figure 7).

As LasR is one of the main players in the QS circuits, inhibition of LasR is associated with subsequent inhibition of virulence factors controlled by lasI/R (protease, hemolysin) and pqs/rhl (pyocyanin and biofilm) (Schuster and Greenberg, 2006). Likewise, ibuprofen binds with LasR by hydrogen bonds at Asp73, Thr75, Thr115, and Ser129 amino acids with significant elimination of pyocyanin, rhamnolipid, and elastase (Dai et al., 2019). Also, 6-Gingerol has formed hydrogen bonding with Trp60, Arg61, and Tyr93 by hydrophobic interactions with Leu40, Tyr47, Ala50, Ala70, Val76, and Ala127 with significant inhibition of QS and virulence factors (Kim et al., 2015).

Moreover, NHQ binds to its receptor PqsR by amino acids Pro238, Thr265, Ala237, Ala168, Phe221, Ile149, Ile236, Pro129, Ala130, Leu197, Leu207, and Leu208. Molecular docking of nanolipoidal $\alpha$-terpineol showed that $\alpha$-terpineol has modulated QS-regulated virulence and biofilm formation in $P$. aeruginosa through competitively binding to LasR, RhlR and Pqs, and QS receptors (Bose et al., 2020).

Hence, the tested compounds CFP, CFPC, and CFPF when docked into the active sites of LasI, LasR and PqsR receptors, they bound with the important amino acids with high ICM scores (Figures 6-8). This indicates that the tested compounds are potential inhibitors of QS and related virulence factors. Data is also supported by phenotypic and molecular studies.

In conclusion, this study explores a new character of CFP to comprise significant inhibition of QS and elimination of associated virulence factors. CFPC and CFPF at 2-8 folds lower concentration than CFP significantly eliminated QS and virulence factors of $P$. aeruginosa including (1) pyocyanin production (2) hemolysin (3) protease and (4) biofilm formation without affecting microbial growth. The interpretation of QSI activity of CFP, CFPC and CFPF was assessed by the molecular model analysis. It may be useful to investigate in vivo activity of CFPC and CFPF in the treatment of $P$. aeruginosa infections.

\section{REFERENCES}

Abagyan, R., Totrov, M., and Kuznetsov, D. (1994). ICM-A New Method for Protein Modeling and Design: Applications to Docking and Structure Prediction From the Distorted Native Conformation. J. Comput. Chem. 15, 488-506. doi: 10.1002/jcc.540150503

Abbas, H. A., Elsherbini, A. M., and Shaldam, M. A. (2017). Repurposing Metformin as a Quorum Sensing Inhibitor in Pseudomonas Aeruginosa. Afr. Health Sci. 17, 808-819. doi: 10.4314/ahs.v17i3.24

Adonizio, A., Kong, K.-F., and Mathee, K. (2008). Inhibition of Quorum SensingControlled Virulence Factor Production in Pseudomonas Aeruginosa by South Florida Plant Extracts. Antimicrob. Agents Chemother. 52, 198-203. doi: 10.1128/AAC.00612-07

Ahmed, T., Pattnaik, S., Khan, M. B., Ampasala, D. R., Busi, S., and Sarma, V. V. (2020). Inhibition of Quorum Sensing-Associated Virulence Factors and Biofilm Formation in Pseudomonas Aeruginosa PAO1 by Mycoleptodiscus Indicus PUTY1. Braz. J. Microbiol. 51, 467-487. doi: 10.1007/s42770-02000235-y

Ahmed, S. A. K. S., Rudden, M., Smyth, T. J., Dooley, J. S. G., Marchant, R., and Banat, I. M. (2019). Natural Quorum Sensing Inhibitors Effectively Downregulate Gene Expression of Pseudomonas Aeruginosa Virulence Factors. Appl. Microbiol. Biotechnol. 103, 3521-3535. doi: 10.1007/s00253019-09618-0

\section{DATA AVAILABILITY STATEMENT}

The original contributions presented in the study are included in the article/Supplementary Material. Further inquiries can be directed to the corresponding author.

\section{AUTHOR CONTRIBUTIONS}

All authors listed have made a substantial, direct, and intellectual contribution to the work, and approved it for publication.

\section{ACKNOWLEDGMENTS}

All appreciation to Prof. Alaa El-Din Ali and Dr. Sherif kolkaila, Department of chemistry, Damanhour University for providing all chemical complexes used in this study. All thanks and appreciation to Prof. Dr., McLean Dept. Biology Texas State University, San Marcos, TX78666, USA for providing Chromobacterium violaceum ATCC 12472 used in the assay of QSI activity performed in this study. All thanks to Prof. to Martin Schuster, Department of Microbiology, Nash Hall, Oregon State University, Corvallis, OR 97331, for providing $P$. aeruginosa $\mathrm{PAO} 1$ and $P$. aeruginosa $\mathrm{PAO}-\mathrm{JP} 2$. All thanks to Dr. Saed Naguib, Mabaret El-Asafra hospital, Alexandria for providing $P$. aeruginosa clinical isolates used in this study.

\section{SUPPLEMENTARY MATERIAL}

The Supplementary Material for this article can be found online at: https://www.frontiersin.org/articles/10.3389/fcimb.2021.716789/ full\#supplementary-material

Aleanizy, F. S., Alqahtani, F. Y., Eltayb, E. K., Alrumikan, N., Almebki, R., Alhossan, A., et al. (2021). Evaluating the Effect of Antibiotics SubInhibitory Dose on Pseudomonas Aeruginosa Quorum Sensing Dependent Virulence and its Phenotypes. Saudi. J. Biol. Sci. 28, 550-559. doi: 10.1016/ j.sjbs.2020.10.040

Bala, A., Kumar, R., and Harjai, K. (2011). Inhibition of Quorum Sensing in Pseudomonas Aeruginosa by Azithromycin and Its Effectiveness in Urinary Tract Infections. J. Med. Microbiol. 60, 300-306. doi: 10.1099/jmm.0.025387-0

Bertani, G. (2004). Lysogeny at Mid-Twentieth Century: P1, P2, and Other Experimental Systems. J. Bacteriol. 186, 595-600. doi: 10.1128/JB.186.3.595600.2004

Bjarnsholt, T., Jensen, P. Ø., Rasmussen, T. B., Christophersen, L., Calum, H., Hentzer, M., et al. (2005). Garlic Blocks Quorum Sensing and Promotes Rapid Clearing of Pulmonary Pseudomonas Aeruginosa Infections. Microbiology 151, 3873-3880. doi: 10.1099/mic.0.27955-0

Borges, A., Simões, M., Todorović, T. R., Filipović, N. R., and García-Sosa, A. T. (2018). Cobalt Complex With Thiazole-Based Ligand as New Pseudomonas Aeruginosa Quorum Quencher, Biofilm Inhibitor and Virulence Attenuator. Molecules 23, 1385. doi: 10.3390/molecules23061385

Bose, S. K., Nirbhavane, P., Batra, M., Chhibber, S., and Harjai, K. (2020). Nanolipoidal $\alpha$-Terpineol Modulates Quorum Sensing Regulated Virulence and Biofilm Formation in Pseudomonas Aeruginosa. Nanomedicine 15, 1743 1760. doi: $10.2217 / \mathrm{nnm}-2020-0134$ 
Bottomley, M. J., Muraglia, E., Bazzo, R., and Carfi, A. (2007). Molecular Insights Into Quorum Sensing in the Human Pathogen Pseudomonas Aeruginosa From the Structure of the Virulence Regulator LasR Bound to Its Autoinducer. J. Biol. Chem. 282, 13592-13600. doi: 10.1074/jbc.M700556200

Burcu Bali, E., Erkan Türkmen, K., Erdönmez, D., and Sağlam, N. (2019). Comparative Study of Inhibitory Potential of Dietary Phytochemicals Against Quorum Sensing Activity of and Biofilm Formation by Chromobacterium Violaceum 12472, and Swimming and Swarming Behaviour of Pseudomonas Aeruginosa PAO1. Food Technol. Biotechnol. 57, 212-221. doi: 10.17113/ftb.57.02.19.5823

Chaverra Daza, K. E., Silva Gómez, E., Moreno Murillo, B. D., and Mayorga Wandurraga, H. (2021). Natural and Enantiopure Alkylglycerols as Antibiofilms Against Clinical Bacterial Isolates and Quorum Sensing Inhibitors of Chromobacterium Violaceum ATCC 12472. Antibiotics 10, 430. doi: 10.3390/antibiotics 10040430

CLSI Clinical and Laboratory Standards Institute (2014). Performance Standards for Antimicrobial Susceptibility Testing: 24 Informational Supplement M100S24 (PA: CLSI, Wayne).

Dai, L., Wu, T., Xiong, Y., Ni, H., Ding, Y., Zhang, W., et al. (2019). IbuprofenMediated Potential Inhibition of Biofilm Development and Quorum Sensing in Pseudomonas Aeruginosa. Life Sci. 237, 116947. doi: 10.1016/j.lfs.2019.116947

Déziel, E., Gopalan, S., Tampakaki, A. P., Lépine, F., Padfield, K. E., Saucier, M., et al. (2005). The Contribution of MvfR to Pseudomonas Aeruginosa Pathogenesis and Quorum Sensing Circuitry Regulation: Multiple Quorum Sensing-Regulated Genes are Modulated Without Affecting lasRI, rhlRI or the Production of N-Acyl-L-Homoserine Lactones. Mol. Microbiol. 55, 998-1014. doi: 10.1111/j.1365-2958.2004.04448.x

Dubern, J.-F., and Diggle, S. P. (2008). Quorum Sensing by 2-Alkyl-4-Quinolones in Pseudomonas Aeruginosa and Other Bacterial Species. Mol. Biosyst. 4, 882888. doi: 10.1039/b803796p

El-Mowafy, S. A., Abd El Galil, K. H., El-Messery, S. M., and Shaaban, M. I. (2014). Aspirin Is an Efficient Inhibitor of Quorum Sensing, Virulence and Toxins in Pseudomonas Aeruginosa. Microb. Pathog. 74, 25-32. doi: 10.1016/ j.micpath.2014.07.008

El-Mowafy, S. A., Abd El Galil, K. H., Habib, E.-S. E., and Shaaban, M. I. (2017). Quorum Sensing Inhibitory Activity of Sub-Inhibitory Concentrations of $\beta$ Lactams. Afr. Health Sci. 17, 199-207. doi: 10.4314/ahs.v17i1.25

El-Shaer, S., Shaaban, M., Barwa, R., and Hassan, R. (2016). Control of Quorum Sensing and Virulence Factors of Pseudomonas Aeruginosa Using Phenylalanine Arginyl $\beta$-Naphthylamide. J. Med. Microbiol. 65, 1194-1204. doi: $10.1099 / \mathrm{jmm} .0 .000327$

Gabr, M. T., El-Gohary, N. S., El-Bendary, E. R., El-Kerdawy, M. M., Ni, N., and Shaaban, M. I. (2015). Synthesis, Antimicrobial, Antiquorum-Sensing and Cytotoxic Activities of New Series of Benzothiazole Derivatives. Chin. Chem. Lett. 26, 1522-1528. doi: 10.1016/j.cclet.2015.09.004

Gabr, M. T., El-Gohary, N. S., El-Bendary, E. R., Ni, N., Shaaban, M. I., and ElKerdawy, M. M. (2018). Microwave-Assisted Synthesis, Antimicrobial, Antiquorum-Sensing and Cytotoxic Activities of a New Series of Isatin- $\beta$ Thiocarbohydrazones. Synth. Commun. 48, 2899-2911. doi: 10.1080/ 00397911.2018.1520889

Garske, L. A., Beatson, S. A., Leech, A. J., Walsh, S. L., and Bell, S. C. (2004). SubInhibitory Concentrations of Ceftazidime and Tobramycin Reduce the Quorum Sensing Signals of Pseudomonas Aeruginosa. Pathology 36, 571575. doi: 10.1080/00313020400011300

Gould, T. A., Schweizer, H. P., and Churchill, M. E. A. (2004). Structure of the Pseudomonas Aeruginosa Acyl-Homoserinelactone Synthase LasI. Mol. Microbiol. 53, 1135-1146. doi: 10.1111/j.1365-2958.2004.04211.x

Gupta, R. K., Setia, S., and Harjai, K. (2011). Expression of Quorum Sensing and Virulence Factors are Interlinked in Pseudomonas Aeruginosa: An In Vitro Approach. Am. J. BioMed. Sci. 3, 116-125. doi: 10.5099/aj110200116

Hassan, R., Shaaban, M. I., Abdel Bar, F. M., El-Mahdy, A. M., and Shokralla, S. (2016). Quorum Sensing Inhibiting Activity of Streptomyces Coelicoflavus Isolated From Soil. Front. Microbiol. 7, 659. doi: 10.3389/fmicb.2016.00659

He, X., Hwang, H., Aker, W. G., Wang, P., Lin, Y., Jiang, X., et al. (2014). Synergistic Combination of Marine Oligosaccharides and Azithromycin Against Pseudomonas Aeruginosa. Microbiol. Res. 169, 759-767. doi: 10.1016/j.micres.2014.01.001
Hentzer, M., and Givskov, M. (2003). Pharmacological Inhibition of Quorum Sensing for the Treatment of Chronic Bacterial Infections. J. Clin. Invest. 112, 1300-1307. doi: 10.1172/JCI20074

Hentzer, M., Riedel, K., Rasmussen, T. B., Heydorn, A., Andersen, J. B., Parsek, M. R., et al. (2002). Inhibition of Quorum Sensing in Pseudomonas Aeruginosa Biofilm Bacteria by a Halogenated Furanone Compound. Microbiology 148, 87-102. doi: 10.1099/00221287-148-1-87

Hnamte, S., Subhaswaraj, P., Ranganathan, S. K., Ampasala, D. R., Muralitharan, G., and Siddhardha, B. (2020). Anti Quorum Sensing and Anti Biofilm Potential of Anogeissus Acuminata and Mallotus Roxburghianus Muell. Against Pseudomonas Aeruginosa PAO1. J. Microbiol. Biotechnol. Food Sci. 9, 1135-1140. doi: 10.15414/jmbfs.2019.8.5.1135-1140

Husain, F. M., and Ahmad, I. (2013). Doxycycline Interferes With Quorum Sensing-Mediated Virulence Factors and Biofilm Formation in GramNegative Bacteria. World J. Microbiol. Biotechnol. 29, 949-957. doi: 10.1007/ s11274-013-1252-1

Ilangovan, A., Fletcher, M., Rampioni, G., Pustelny, C., Rumbaugh, K., Heeb, S., et al. (2013). Structural Basis for Native Agonist and Synthetic Inhibitor Recognition by the Pseudomonas Aeruginosa Quorum Sensing Regulator PqsR (MvfR). PloS Pathog. 9, e1003508. doi: 10.1371/journal.ppat.1003508

Kim, H.-S., Lee, S.-H., Byun, Y., and Park, H.-D. (2015). 6-Gingerol Reduces Pseudomonas Aeruginosa Biofilm Formation and Virulence via Quorum Sensing Inhibition. Sci. Rep. 5, 1-11. doi: 10.1038/srep08656

Latifi, A., Foglino, M., Tanaka, K., Williams, P., and Lazdunski, A. (1996). A Hierarchical Quorum-Sensing Cascade in Pseudomonas Aeruginosa Links the Transcriptional Activators LasR and RhIR (VsmR) to Expression of the Stationary-Phase Sigma Factor RpoS. Mol. Microbiol. 21, 1137-1146. doi: 10.1046/j.1365-2958.1996.00063.x

Lee, J., and Zhang, L. (2015). The Hierarchy Quorum Sensing Network in Pseudomonas Aeruginosa. Protein Cell 6, 26-41. doi: 10.1007/s13238-0140100-x

Masoud, M. S., Ali, A. E., Elasala, G. S., and Kolkaila, S. H. (2017). Spectroscopic Studies and Thermal Analysis on Cefoperazone Metal Complexes. J. Chem. Pharm. Res. 9, 171-179. doi: 10.1016/j.heliyon.2019.e02912

McClean, K. H., Winson, M. K., Fish, L., Taylor, A., Chhabra, S. R., Camara, M., et al. (1997). Quorum Sensing and Chromobacterium Violaceum: Exploitation of Violacein Production and Inhibition for the Detection of N-Acylhomoserine Lactones. Microbiology 143, 3703-3711. doi: 10.1099/00221287-143-12-3703

McLean, R. J. C., Pierson, L. S.III, and Fuqua, C. (2004). A Simple Screening Protocol for the Identification of Quorum Signal Antagonists. J. Microbiol. Methods 58, 351-360. doi: 10.1016/j.mimet.2004.04.016

Mion, S., Carriot, N., Lopez, J., Plener, L., Ortalo-Magné, A., Chabrière, E., et al. (2021). Disrupting Quorum Sensing Alters Social Interactions in Chromobacterium Violaceum. NPJ Biofilms. Microbiomes. 7, 1-16. doi: 10.1038/s41522-021-00211-w

Monterrosa, M. A., Galindo, J. F., Lorduy, J. V., Alí-Torres, J., and Vivas-Reyes, R. (2019). The Role of LasR Active Site Amino Acids in the Interaction With the Acyl Homoserine Lactones (AHLs) Analogues: A Computational Study. J. Mol. Graph. Model. 86, 113-124. doi: 10.1016/j.jmgm.2018.10.014

Morohoshi, T., Kato, M., Fukamachi, K., Kato, N., and Ikeda, T. (2008). NAcylhomoserine Lactone Regulates Violacein Production in Chromobacterium Violaceum Type Strain ATCC 12472. FEMS Microbiol. Lett. 279, 124-130. doi: 10.1111/j.1574-6968.2007.01016.x

Musthafa, K. S., Sivamaruthi, B. S., Pandian, S. K., and Ravi, A. V. (2012). Quorum Sensing Inhibition in Pseudomonas Aeruginosa PAO1 by Antagonistic Compound Phenylacetic Acid. Curr. Microbiol. 65, 475-480. doi: 10.1007/ s00284-012-0181-9

Nalca, Y., Jänsch, L., Bredenbruch, F., Geffers, R., Buer, J., and Häussler, S. (2006). Quorum-Sensing Antagonistic Activities of Azithromycin in Pseudomonas Aeruginosa PAO1: A Global Approach. Antimicrob. Agents Chemother. 50, 1680-1688. doi: 10.1128/AAC.50.5.1680-1688.2006

Parsek, M. R., and Greenberg, E. P. (2000). Acyl-Homoserine Lactone Quorum Sensing in Gram-Negative Bacteria: A Signaling Mechanism Involved in Associations With Higher Organisms. Proc. Natl. Acad. Sci. 97, 8789-8793. doi: $10.1073 /$ pnas. 97.16 .8789

Pearson, J. P., Pesci, E. C., and Iglewski, B. H. (1997). Roles of Pseudomonas Aeruginosa Las and Rhl Quorum-Sensing Systems in Control of Elastase and 
Rhamnolipid Biosynthesis Genes. J. Bacteriol. 179, 5756-5767. doi: 10.1128/ jb.179.18.5756-5767.1997

Pesci, E. C., Pearson, J. P., Seed, P. C., and Iglewski, B. H. (1997). Regulation of Las and Rhl Quorum Sensing in Pseudomonas Aeruginosa. J. Bacteriol. 179, 31273132. doi: $10.1128 /$ jb.179.10.3127-3132.1997

Pessi, G., Williams, F., Hindle, Z., Heurlier, K., Holden, M. T. G., Cámara, M., et al. (2001). The Global Posttranscriptional Regulator RsmA Modulates Production of Virulence Determinants and N-Acylhomoserine Lactones in Pseudomonas Aeruginosa. J. Bacteriol. 183, 6676-6683. doi: 10.1128/JB.183.22.66766683.2001

Prateeksha, R., V, C., Das, A. K., Barik, S. K., and Singh, B. N. (2019). ZnO/ curcumin Nanocomposites for Enhanced Inhibition of Pseudomonas Aeruginosa Virulence via LasR-RhlR Quorum Sensing Systems. Mol. Pharm. 16, 3399-3413. doi: 10.1021/acs.molpharmaceut.9b00179

Proctor, C. R., McCarron, P. A., and Ternan, N. G. (2020). Furanone QuorumSensing Inhibitors With Potential as Novel Therapeutics Against Pseudomonas Aeruginosa. J. Med. Microbiol. 69, 195-206. doi: 10.1099/jmm.0.001144

Rampioni, G., Falcone, M., Heeb, S., Frangipani, E., Fletcher, M. P., Dubern, J.-F., et al. (2016). Unravelling the Genome-Wide Contributions of Specific 2-Alkyl4-Quinolones and PqsE to Quorum Sensing in Pseudomonas Aeruginosa. PloS Pathog. 12, e1006029. doi: 10.1371/journal.ppat.1006029

Rekha, P. D., Vasavi, H. S., Vipin, C., Saptami, K., and Arun, A. B. (2017). A Medicinal Herb Cassia Alata Attenuates Quorum Sensing in Chromobacterium Violaceum and Pseudomonas Aeruginosa. Lett. Appl. Microbiol. 64, 231-238. doi: 10.1111/lam.12710

Rossignol, G., Merieau, A., Guerillon, J., Veron, W., Lesouhaitier, O., Feuilloley, M. G. J., et al. (2008). Involvement of a Phospholipase C in the Hemolytic Activity of a Clinical Strain of Pseudomonas Fluorescens. BMC Microbiol. 8, 1-14. doi: 10.1186/1471-2180-8-189

Saleh, M. M., Refa't A, S., Latif, H. K. A., Abbas, H. A., and Askoura, M. (2019). Zinc Oxide Nanoparticles Inhibits Quorum Sensing and Virulence in Pseudomonas Aeruginosa. Afr. Health Sci. 19, 2043-2055. doi: 10.4314/ahs.v19i2.28

Saurav, K., Bar-Shalom, R., Haber, M., Burgsdorf, I., Oliviero, G., Costantino, V., et al. (2016). In Search of Alternative Antibiotic Drugs: Quorum-Quenching Activity in Sponges and Their Bacterial Isolates. Front. Microbiol. 7, 416. doi: 10.3389/fmicb.2016.00416

Schuster, M., and Greenberg, E. P. (2006). A Network of Networks: QuorumSensing Gene Regulation in Pseudomonas Aeruginosa. Int. J. Med. Microbiol. 296, 73-81. doi: 10.1016/j.jimm.2006.01.036

Shaaban, M., Elgaml, A., and Habib, E.-S. E. (2019). Biotechnological Applications of Quorum Sensing Inhibition as Novel Therapeutic Strategies for Multidrug Resistant Pathogens. Microb. Pathog. 127, 138-143. doi: 10.1016/ j.micpath.2018.11.043

Skindersoe, M. E., Alhede, M., Phipps, R., Yang, L., Jensen, P. O., Rasmussen, T. B., et al. (2008). Effects of Antibiotics on Quorum Sensing in Pseudomonas Aeruginosa. Antimicrob. Agents Chemother. 52, 3648-3663. doi: 10.1128/ AAC.01230-07

Standards Australia (1995). Water Microbiology-Heterotrophic Colony Count Methods - Pour Plate Method Using Plate Count Agar. AS 4276.3.1, Committee FT/20, Water Microbiology. Homebush, Australia: Council of Standards Australia.
Swatton, J. E., Davenport, P. W., Maunders, E. A., Griffin, J. L., Lilley, K. S., and Welch, M. (2016). Impact of Azithromycin on the Quorum Sensing-Controlled Proteome of Pseudomonas Aeruginosa. PloS One 11, e0147698. doi: 10.1371/ journal.pone. 0147698

Tapia-Rodriguez, M. R., Bernal-Mercado, A. T., Gutierrez-Pacheco, M. M., Vazquez-Armenta, F. J., Hernandez-Mendoza, A., Gonzalez-Aguilar, G. A., et al. (2019). Virulence of Pseudomonas Aeruginosa Exposed to Carvacrol: Alterations of the Quorum Sensing at Enzymatic and Gene Levels. J. Cell Commun. Signal. 13, 531-537. doi: 10.1007/s12079-019-00516-8

Tateda, K., Comte, R., Pechere, J.-C., Köhler, T., Yamaguchi, K., and Van Delden, C. (2001). Azithromycin Inhibits Quorum Sensing in Pseudomonas Aeruginosa. Antimicrob. Agents Chemother. 45, 1930-1933. doi: 10.1128/ AAC.45.6.1930-1933.2001

Thornton, J. M., Walker, J. M., Sundarasivarao, P. Y. K., Spur, B. W., Rodriguez, A., and Yin, K. (2020). Lipoxin A4 Promotes Reduction and Antibiotic Efficacy Against Pseudomonas Aeruginosa Biofilm. Prostaglandins Other. Lipid Mediat. 152, 106505. doi: 10.1016/j.prostaglandins.2020.106505

Turkina, M. V., and Vikström, E. (2019). Bacteria-Host Crosstalk: Sensing of the Quorum in the Context of Pseudomonas Aeruginosa Infections. J. Innate. Immun. 11, 263-279. doi: 10.1159/000494069

Tyagi, P., Singh, M., Kumari, H., Kumari, A., and Mukhopadhyay, K. (2015). Bactericidal Activity of Curcumin I Is Associated With Damaging of Bacterial Membrane. PloS One 10, e0121313. doi: 10.1371/journal.pone.0121313

Vasavi, H. S., Arun, A. B., and Rekha, P. (2014). Anti-Quorum Sensing Activity of Psidium Guajava L. Flavonoids Against Chromobacterium Violaceum and Pseudomonas Aeruginosa PAO1. Microbiol. Immunol. 58, 286-293. doi: 10.1111/1348-0421.12150

Wagner, V. E., Bushnell, D., Passador, L., Brooks, A. I., and Iglewski, B. H. (2003). Microarray Analysis of Pseudomonas Aeruginosa Quorum-Sensing Regulons: Effects of Growth Phase and Environment. J. Bacteriol. 185, 2080-2095. doi: 10.1128/JB.185.7.2080-2095.2003

Conflict of Interest: The authors declare that the research was conducted in the absence of any commercial or financial relationships that could be construed as a potential conflict of interest.

Publisher's Note: All claims expressed in this article are solely those of the authors and do not necessarily represent those of their affiliated organizations, or those of the publisher, the editors and the reviewers. Any product that may be evaluated in this article, or claim that may be made by its manufacturer, is not guaranteed or endorsed by the publisher.

Copyright $\odot 2021$ Naga, El-Badan, Rateb, Ghanem and Shaaban. This is an openaccess article distributed under the terms of the Creative Commons Attribution License (CC BY). The use, distribution or reproduction in other forums is permitted, provided the original author(s) and the copyright owner(s) are credited and that the original publication in this journal is cited, in accordance with accepted academic practice. No use, distribution or reproduction is permitted which does not comply with these terms. 